\title{
Testing SU(3) flavor symmetry in semileptonic and two-body nonleptonic decays of hyperons
}

\author{
Ru-Min Wang $\oplus^{1, *}$ Mao-Zhi Yang, ${ }^{2, \dagger}$ Hai-Bo Li, ${ }^{3,4, \ddagger}$ and Xiao-Dong Cheng $\oplus^{5, \S}$ \\ ${ }^{1}$ College of Physics and Communication Electronics, Jiangxi Normal University, \\ NanChang, Jiangxi 330022, China \\ ${ }^{2}$ School of Physics, Nankai University, Tianjin 300071, China \\ ${ }^{3}$ Institute of High Energy Physics, Beijing 100049, China \\ ${ }^{4}$ University of Chinese Academy of Sciences, Beijing 100049, China \\ ${ }^{5}$ College of Physics and Electronic Engineering, Xinyang Normal University, \\ Xinyang, Henan 464000, China
}

(Received 26 June 2019; published 14 October 2019)

\begin{abstract}
The semileptonic decays and two-body nonleptonic decays of a light baryon octet $\left(T_{8}\right)$ and a decuplet $\left(T_{10}\right)$ consisting of light $u, d, s$ quarks are studied with the $\mathrm{SU}(3)$ flavor symmetry. We obtain the amplitude relations between different decay modes by the $\mathrm{SU}(3)$ irreducible representation approach, and we then predict relevant branching ratios by presenting experimental data within $1 \sigma$ error. We find that the predictions for all branching ratios except $\mathcal{B}\left(\Xi \rightarrow \Lambda^{0} \pi\right)$ and $\mathcal{B}\left(\Xi^{*} \rightarrow \Xi \pi\right)$ are in good agreement with present experimental data, which implies that the neglected $C_{+}$terms or SU(3) breaking effects might contribute on the order of a few percent in $\Xi \rightarrow \Lambda^{0} \pi$ and $\Xi^{*} \rightarrow \Xi \pi$ weak decays. We predict that $\mathcal{B}\left(\Xi^{-} \rightarrow \Sigma^{0} \mu^{-} \bar{\nu}_{\mu}\right)=(1.13 \pm 0.08) \times 10^{-6}$, $\mathcal{B}\left(\Xi^{-} \rightarrow \Lambda^{0} \mu^{-} \bar{\nu}_{\mu}\right)=(1.58 \pm 0.04) \times 10^{-4}, \mathcal{B}\left(\Omega^{-} \rightarrow \Xi^{0} \mu^{-} \bar{\nu}_{\mu}\right)=(3.7 \pm 1.8) \times 10^{-3}, \mathcal{B}\left(\Sigma^{-} \rightarrow \Sigma^{0} e^{-} \bar{\nu}_{e}\right)=$ $(1.35 \pm 0.28) \times 10^{-10}$, and $\mathcal{B}\left(\Xi^{-} \rightarrow \Xi^{0} e^{-} \bar{\nu}_{e}\right)=(4.2 \pm 2.4) \times 10^{-10}$. We also study $T_{10} \rightarrow T_{8} P_{8}$ weak, electromagnetic, or strong decays. Some of these decay modes could be observed by BESIII, LHCb, and other experiments in the near future. Because of the very small lifetimes of $\Sigma^{0}, \Xi^{* 0,-}, \Sigma^{* 0,-}$, and $\Delta^{0,-}$, the branching ratios of these baryon weak decays are only on the order of $\mathcal{O}\left(10^{-20}-10^{-13}\right)$, which is too small to be reached in current experiments. The longitudinal branching ratios of $T_{8 A} \rightarrow T_{8 B} \ell^{-} \bar{\nu}_{\ell}(\ell=\mu, e)$ decays are also given.
\end{abstract}

DOI: 10.1103/PhysRevD.100.076008

\section{INTRODUCTION}

A lot of semileptonic decays and two-body nonleptonic decays of light octet baryons (such as $\Xi^{-} \rightarrow \Sigma^{0} e^{-} \bar{\nu}_{e}$, $\Xi^{-} \rightarrow \Lambda^{0} \ell^{-} \bar{\nu}_{\ell}, \quad \Xi^{0} \rightarrow \Sigma^{+} \ell^{-} \bar{\nu}_{\ell}, \Lambda^{0} \rightarrow p \ell^{-} \bar{\nu}_{\ell}, \Sigma^{-} \rightarrow n \ell^{-} \bar{\nu}_{\ell}$, $\Sigma^{-} \rightarrow \Lambda^{0} e^{-} \bar{\nu}_{e}, \Sigma^{+} \rightarrow \Lambda^{0} e^{+} \nu_{e}, n \rightarrow p e^{-} \bar{\nu}_{e}, \Sigma^{+} \rightarrow p \pi^{0}, \Sigma^{+} \rightarrow$ $n \pi^{+}, \Sigma^{-} \rightarrow n \pi^{-}, \Lambda^{0} \rightarrow p \pi^{-}, \Lambda^{0} \rightarrow n \pi^{0}, \Xi^{-} \rightarrow \Lambda^{0} \pi^{-}$, and $\Xi^{0} \rightarrow \Lambda^{0} \pi^{0}$ ) and a few light decuplet baryon decays (such as $\Omega^{-} \rightarrow \Xi^{0} e^{-} \bar{\nu}_{e}, \Xi^{0} \pi^{-}, \Xi^{-} \pi^{0}$, and $\Lambda^{0} K^{-}$) were measured a long time ago by SPEC, HBC, OSPK, etc., [1]. The sensitivity for measurements of $\Lambda, \Sigma, \Xi$, and $\Omega$ hyperon decays is now in the range of $10^{-5}-10^{-8}$ at BESIII [2-5], and these hyperons have also been produced copiously during the

\footnotetext{
ruminwang@sina.com

†yangmz@nankai.edu.cn

tihb@ihep.ac.cn

${ }^{\S}$ chengxd@mails.ccnu.edu.cn
}

Published by the American Physical Society under the terms of the Creative Commons Attribution 4.0 International license. Further distribution of this work must maintain attribution to the author(s) and the published article's title, journal citation, and DOI. Funded by SCOAP ${ }^{3}$.
LHCb experiment [6,7]. Besides confirming information obtained earlier by SPEC, HBC, OSPK, etc., the BESIII and $\mathrm{LHCb}$ experiments will provide new information on light baryon decays. The precise measurements of these decays are of great importance in determining the $V-A$ structure and quark-flavor mixing of charged current weak interactions [8-10] as well as probing the nonstandard charged current interactions $[11,12]$.

Theoretically, the factorization does not work well for $s$, $d$ quark decays since $s, d$ quarks are very light and cannot use the heavy quark expansion. There is no reliable method to calculate these decay matrix elements at present. In the absence of reliable calculations, the symmetry analysis can provide very useful information about the decays. SU(3) flavor symmetry is one of the symmetries which have attracted a lot of attention. The SU(3) flavor symmetry approach, which is independent of the detailed dynamics, offers an opportunity to relate different decay modes. Nevertheless, it cannot determine the size of the amplitudes by itself. However, if experimental data are enough, one may use the data to extract the $\mathrm{SU}(3)$ irreducible amplitudes, which can be viewed as predictions based on symmetry. There are two popular approaches to SU(3) 
flavor symmetry. One is to construct the SU(3) irreducible representation amplitude by decomposing an effective Hamiltonian. The other method is the topological diagram approach, where decay amplitudes are represented by connecting quark line flows in different ways and then relating them through the $\mathrm{SU}(3)$ symmetry.

The SU(3) flavor symmetry works well in heavy hadron decays-for instance, $b$-hadron decays [13-26] and $c$ hadron decays [27-43]. The experimental data of some semileptonic hyperon decays are well explained by the Cabibbo theory [10], which assumes that the SU(3) symmetry breaking effects are neglected. The SU(3) flavor symmetry breaking effects are also studied in the hyperon beta decays [44-47], where it is found that the SU(3) symmetry breaking effects in these decays are small. In this paper, we will systematically study $T_{8,10} \rightarrow T_{8} \ell^{-} \bar{\nu}_{\ell}$ and $T_{8,10} \rightarrow T_{8} P$ decays using the SU(3) irreducible representation approach (IRA). We will first construct the SU(3) irreducible representation amplitudes for different kinds of $T_{8}$ and $T_{10}$ decays, second obtain the decay amplitude relations between different decay modes, then use the

$$
T_{10}=\frac{1}{\sqrt{3}}\left(\left(\begin{array}{ccc}
\sqrt{3} \Delta^{++} & \Delta^{+} & \Sigma^{*+} \\
\Delta^{+} & \Delta^{0} & \frac{\Sigma^{* 0}}{\sqrt{2}} \\
\Sigma^{*+} & \frac{\Sigma^{* 0}}{\sqrt{2}} & \Xi^{* 0}
\end{array}\right),\left(\begin{array}{c}
\Delta^{+} \\
\Delta^{0} \\
\frac{\Sigma^{* 0}}{\sqrt{2}}
\end{array}\right.\right.
$$

In this section, we focus on $\Delta S=0$ and $\Delta S=1$ semileptonic decays of hyperons, which decay through $d \rightarrow u e^{-} \bar{\nu}_{e}$ and $s \rightarrow u \ell^{-} \bar{\nu}_{\ell}$ transitions, respectively. Since $\Delta S=2$ semileptonic decays are forbidden, we will not study them in this work.

\section{A. $T_{8 A} \rightarrow T_{8 B} \ell^{-} \bar{\nu}_{\ell}$ semileptonic decays}

\section{Theoretical framework}

In the Standard Model (SM), the Feynman diagram for $T_{8 A} \rightarrow T_{8 B} \ell^{-} \bar{\nu}_{\ell}$ decays is shown in Fig. 1, and the amplitudes of $T_{8 A} \rightarrow T_{8 B} \ell^{-} \bar{\nu}_{\ell}$ can be written as [48]

$$
\begin{aligned}
\mathcal{A} & \left(T_{8 A} \rightarrow T_{8 B} \ell^{-} \bar{\nu}_{\ell}\right) \\
& =\sum_{\lambda_{W}, \lambda_{W}^{\prime}= \pm 1,0, t} \frac{G_{F}}{2 \sqrt{2}} H_{\lambda_{B} \lambda_{W}} \bar{u}_{\ell} \gamma_{\beta}\left(1-\gamma_{5}\right) v_{\nu} \epsilon^{* \beta}\left(\lambda_{W}^{\prime}\right) g_{\lambda_{W} \lambda_{W}^{\prime}},
\end{aligned}
$$

where $G_{F}$ is the Fermi constant, $\lambda_{B}$ and $\lambda_{W}$ are the helicity components of the $T_{8 B}$ baryon and the virtual $W$ boson in the $T_{8 A}$ rest frame, $\lambda_{W}^{\prime}$ is the helicity component of the virtual $W$ boson in the $\ell \nu_{\ell}$ rest frame, $\bar{u}_{\ell}$ and $v_{\nu}$ are Dirac spinors, and $\epsilon^{* \beta}\left(\lambda_{W}^{\prime}\right)$ are the polarization vectors of $W$ in the $\ell \nu_{\ell}$ rest frame. The helicity amplitudes $H_{\lambda_{B} \lambda_{W}}$ are available data to extract the SU(3) irreducible amplitudes, and finally predict the not-yet-measured modes for further tests in experiments.

This paper is organized as follows. In Sec. II, the semileptonic weak decays of the $T_{8,10}$ hyperons are studied. In Sec. III, we will explore the two-body nonleptonic decays of hyperons which are through weak interaction, electromagnetic, or strong interaction. Our conclusions are given in Sec. IV.

\section{SEMILEPTONIC DECAYS OF HYPERONS}

The light baryons $T_{8}\left(T_{10}\right)$, which are octets (decuplets) under the $\mathrm{SU}(3)$ flavor symmetry of $u, d, s$ quarks, can be written as

$$
T_{8}=\left(\begin{array}{ccc}
\frac{\Lambda^{0}}{\sqrt{6}}+\frac{\Sigma^{0}}{\sqrt{2}} & \Sigma^{+} & p \\
\Sigma^{-} & \frac{\Lambda^{0}}{\sqrt{6}}-\frac{\Sigma^{0}}{\sqrt{2}} & n \\
\Xi^{-} & \Xi^{0} & -\frac{2 \Lambda^{0}}{\sqrt{6}}
\end{array}\right),
$$

$$
\left.\left.\begin{array}{cc}
\Delta^{0} & \frac{\Sigma^{* 0}}{\sqrt{2}} \\
\sqrt{3} \Delta^{-} & \Sigma^{*-} \\
\Sigma^{*-} & \Xi^{*-}
\end{array}\right),\left(\begin{array}{ccc}
\Sigma^{*+} & \frac{\Sigma^{* 0}}{\sqrt{2}} & \Xi^{* 0} \\
\frac{\Sigma^{* 0}}{\sqrt{2}} & \Sigma^{*-} & \Xi^{*-} \\
\Xi^{*-} & \Xi^{*-} & \sqrt{3} \Omega^{-}
\end{array}\right)\right) .
$$

$$
\begin{aligned}
& H_{\lambda_{B} \lambda_{W}}=H_{\lambda_{B} \lambda_{W}}^{V}-H_{\lambda_{B} \lambda_{W}}^{A}, \\
& H_{\lambda_{B} \lambda_{W}}^{V(A)}=\left\langle T_{8 B}\left|J_{\mu}^{V(A)}\right| T_{8 A}\right\rangle \epsilon^{\mu}\left(\lambda_{W}\right),
\end{aligned}
$$

where $J_{\mu}^{V(A)}$ is the vector (axial vector) current, and $\epsilon^{\mu}\left(\lambda_{W}\right)$ are the polarization vectors of $W$ in the $T_{8 A}$ rest frame.

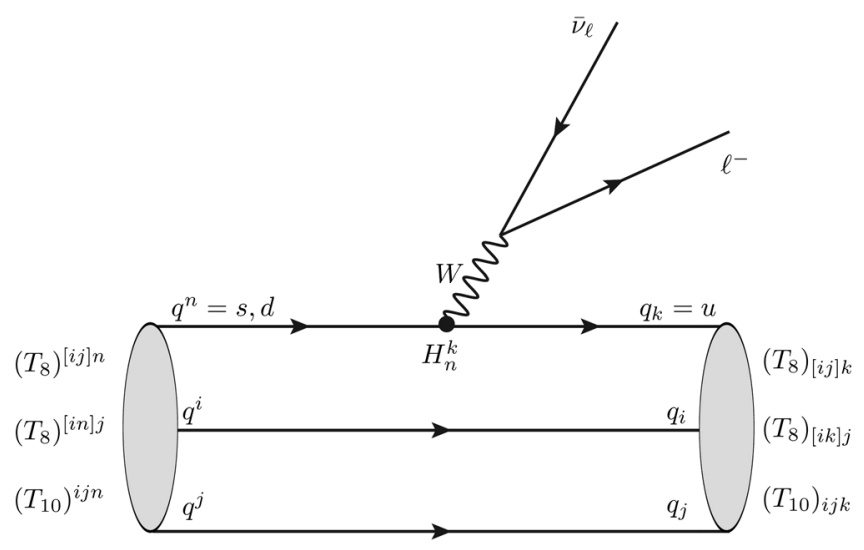

FIG. 1. Feynman diagram for semileptonic weak decays $T_{8,10} \rightarrow T_{8} \ell \bar{\nu}_{\ell}$. 
TABLE I. The helicity amplitudes $H_{\lambda_{B} \lambda_{W}}^{V(A)}$ of $T_{8 A} \rightarrow T_{8 B} \ell^{-} \bar{\nu}_{\ell}$ decays.

\begin{tabular}{lcc}
\hline \hline$H_{\lambda_{B} \lambda_{W}}^{V(A)}$ & SU(3) IRA amplitudes & Reparametrization \\
\hline$s \rightarrow u \ell^{-} \bar{\nu}_{\ell}:$ & & $-A_{31}$ \\
$\sqrt{2} H\left(\Xi^{-} \rightarrow \Sigma^{0} \ell^{-} \bar{\nu}_{\ell}\right)$ & $-\left(a_{31}+a_{32}+a_{33}+a_{35}\right)$ & $A_{31}+2 A_{32}$ \\
$\sqrt{6} H\left(\Xi^{-} \rightarrow \Lambda^{0} \ell^{-} \bar{\nu}_{\ell}\right)$ & $a_{31}+a_{32}+a_{33}+2 a_{34}-a_{35}$ & $A_{31}$ \\
$H\left(\Xi^{0} \rightarrow \Sigma^{+} \ell^{-} \bar{\nu}_{\ell}\right)$ & $a_{31}+a_{32}+a_{33}+a_{35}$ & $-\left(2 A_{31}+A_{32}\right)$ \\
$\sqrt{6} H\left(\Lambda^{0} \rightarrow p \ell^{-} \bar{\nu}_{\ell}\right)$ & $-\left(2 a_{31}+2 a_{32}+2 a_{33}+a_{34}+a_{35}\right)$ & $A_{32}$ \\
$\sqrt{2} H\left(\Sigma^{0} \rightarrow p \ell^{-} \bar{\nu}_{\ell}\right)$ & $a_{34}-a_{35}$ & $-A_{32}$ \\
$H\left(\Sigma^{-} \rightarrow n \ell^{-} \bar{\nu}_{\ell}\right)$ & $-\left(a_{34}-a_{35}\right)$ & $-\left(2 A_{21}-\bar{A}_{22}\right)$ \\
$d \rightarrow u e^{-} \bar{\nu}_{e}:$ & & $\bar{A}_{22}$ \\
$\sqrt{2} H\left(\Sigma^{-} \rightarrow \Sigma^{0} e^{-} \bar{\nu}_{e}\right)$ & $-\left(a_{21}+a_{22}+a_{23}+a_{24}\right)$ & $\bar{A}_{22}$ \\
$\sqrt{6} H\left(\Sigma^{-} \rightarrow \Lambda^{0} e^{-} \bar{\nu}_{e}\right)$ & $a_{21}+a_{22}+a_{23}-a_{24}+2 a_{22}$ & $\bar{A}_{22}$ \\
$\sqrt{2} H\left(\Sigma^{0} \rightarrow \Sigma^{+} e^{-} \bar{\nu}_{e}\right)$ & $a_{21}+a_{22}+a_{23}+a_{24}$ & $\bar{A}_{22}-A_{21}$ \\
$\sqrt{6} H\left(\Sigma^{+} \rightarrow \Lambda^{0} e^{+} \nu_{e}\right)$ & $a_{21}+a_{22}+a_{23}-a_{24}+2 a_{22}$ & $A_{21}$ \\
$H\left(\Xi^{-} \rightarrow \Xi^{0} e^{-} \bar{\nu}_{e}\right)$ & $-a_{22}$ & \\
$H\left(n \rightarrow p e^{-} \bar{\nu}_{e}\right)$ & $a_{21}+a_{22}+a_{23}+a_{22}$ & \\
\hline \hline
\end{tabular}

In terms of the SU(3) IRA, the vector (axial vector) helicity amplitudes $H_{\lambda_{B} \lambda_{W}}^{V(A)}$ can be parametrized as

$$
\begin{aligned}
H_{\lambda_{B} \lambda_{W}}^{V(A)}= & a_{n 1} H_{n}^{k}\left(T_{8}\right)^{[i j] n}\left(T_{8}\right)_{[i j] k}+a_{n 2} H_{n}^{k}\left(T_{8}\right)^{[i j] n}\left(T_{8}\right)_{[i k] j} \\
& +a_{n 3} H_{n}^{k}\left(T_{8}\right)^{[i n] j}\left(T_{8}\right)_{[i j] k}+a_{n 4} H_{n}^{k}\left(T_{8}\right)^{[i n] j}\left(T_{8}\right)_{[i k] j} \\
& +a_{n 5} H_{n}^{k}\left(T_{8}\right)^{[i n] j}\left(T_{8}\right)_{[k j] i}
\end{aligned}
$$

where $H_{n}^{k}=V_{q_{k} q_{n}}$ is the Cabibbo-Kobayashi-Maskawa $(\mathrm{CKM})$ matrix element, $a_{n i} \equiv\left(a_{n i}\right)_{\lambda_{B} \lambda_{W}}^{V(A)}\left(q^{2}\right)$ are the nonperturbative coefficients, and $n=2(3)$ for $q_{n}=d(s)$. The SU(3) IRA helicity amplitudes $H_{\lambda_{B} \lambda_{W}}^{V(A)}$ of $T_{8 A} \rightarrow$ $T_{8 B} \ell^{-} \bar{\nu}_{\ell}$ decays are listed in the second column of Table I. And the helicity amplitudes can be simplified by the redefinitions

$$
\begin{aligned}
& A_{n 1}=a_{n 1}+a_{n 2}+a_{n 3}+a_{n 5}, \\
& A_{n 2}=a_{n 4}-a_{n 5} .
\end{aligned}
$$

For convenience, we set $\bar{A}_{22}=A_{21}-A_{22}$ to replace $A_{22}$ for the $d \rightarrow u \ell^{-} \bar{\nu}_{\ell}$ transition. The reparametrization results are given in the last column of Table I, in which we can easily see the helicity amplitude relations between different decay modes.

In addition, the helicity amplitudes can also be written in terms of the $T_{8 A} \rightarrow T_{8 B}$ form factors

$$
\begin{aligned}
& H_{\frac{1}{2} 0}^{V}=\frac{\sqrt{Q_{-}}}{\sqrt{q^{2}}}\left[\left(m_{A}+m_{B}\right) f_{1}\left(q^{2}\right)-q^{2} f_{2}\left(q^{2}\right)\right], \\
& H_{\frac{1}{2} 0}^{A}=\frac{\sqrt{Q_{+}}}{\sqrt{q^{2}}}\left[\left(m_{A}-m_{B}\right) g_{1}\left(q^{2}\right)+q^{2} g_{2}\left(q^{2}\right)\right], \\
& H_{\frac{1}{2} 1}^{V}=\sqrt{2 Q_{-}}\left[-f_{1}\left(q^{2}\right)+\left(m_{A}+m_{B}\right) f_{2}\left(q^{2}\right)\right], \\
& H_{\frac{1}{2} 1}^{A}=\sqrt{2 Q_{+}}\left[-g_{1}\left(q^{2}\right)-\left(m_{A}-m_{B}\right) g_{2}\left(q^{2}\right)\right], \\
& H_{\frac{1}{2} t}^{V}=\frac{\sqrt{Q_{+}}}{\sqrt{q^{2}}}\left[\left(m_{A}-m_{B}\right) f_{1}\left(q^{2}\right)+q^{2} f_{3}\left(q^{2}\right)\right], \\
& H_{\frac{1}{2} t}^{A}=\frac{\sqrt{Q_{-}}}{\sqrt{q^{2}}}\left[\left(m_{A}+m_{B}\right) g_{1}\left(q^{2}\right)-q^{2} g_{3}\left(q^{2}\right)\right],
\end{aligned}
$$

where $m_{A, B}$ is the mass of $T_{8 A, 8 B}$, the momentum transfer $q=p_{A}-p_{B}, \quad Q_{ \pm}=\left(m_{A} \pm m_{B}\right)^{2}-q^{2}$ with $m_{\ell}^{2} \leq q^{2} \leq$ $\left(m_{A}^{2}-m_{B}^{2}\right)$, and the form factors $f_{i}\left(q^{2}\right)$ and $g_{i}\left(q^{2}\right)$ are defined as [26]

$$
\begin{aligned}
& \left\langle T_{8 B}\left(p_{B}, \lambda_{B}\right)\left|\bar{c} \gamma_{\mu} b\right| T_{8 A}\left(p_{A}, \lambda_{A}\right)\right\rangle \\
& =\bar{u}_{B}\left(p_{B}, \lambda_{B}\right)\left[f_{1}\left(q^{2}\right) \gamma_{\mu}+i f_{2}\left(q^{2}\right) \sigma_{\mu \nu} q^{\nu}\right. \\
& \left.\quad+f_{3}\left(q^{2}\right) q_{\mu}\right] u_{A}\left(p_{A}, \lambda_{A}\right), \\
& \left\langle T_{8 B}\left(p_{B}, \lambda_{B}\right)\left|\bar{c} \gamma_{\mu} \gamma^{5} b\right| T_{8 A}\left(p_{A}, \lambda_{A}\right)\right\rangle \\
& =\bar{u}_{B}\left(p_{B}, \lambda_{B}\right)\left[g_{1}\left(q^{2}\right) \gamma_{\mu}+i g_{2}\left(q^{2}\right) \sigma_{\mu \nu} q^{\nu}\right. \\
& \left.\quad+g_{3}\left(q^{2}\right) q_{\mu}\right] \gamma_{5} u_{A}\left(p_{A}, \lambda_{A}\right) .
\end{aligned}
$$

Either from parity or from explicit calculation, we have the relations $H_{-\lambda_{2}-\lambda_{1}}^{V}=H_{\lambda_{2} \lambda_{1}}^{V}, H_{-\lambda_{2}-\lambda_{1}}^{A}=-H_{\lambda_{2} \lambda_{1}}^{A}$. Note that 
TABLE II. The form factor ratios $g_{1}(0) / f_{1}(0)$ and $f_{2}(0) / f_{1}(0)$ come from PDG2018 [1] unless otherwise specified.

\begin{tabular}{lcc}
\hline \hline Decay modes & $g_{1}(0) / f_{1}(0)$ & $f_{2}(0) / f_{1}(0)$ \\
\hline$\Xi^{-} \rightarrow \Sigma^{0} \ell^{-} \bar{\nu}_{\ell}$ & $1.22 \pm 0.05^{\mathrm{a}}$ & $2.609^{\mathrm{b}}$ \\
$\Xi^{-} \rightarrow \Lambda^{0} \ell^{-} \bar{\nu}_{\ell}$ & $0.25 \pm 0.05$ & $0.085^{\mathrm{b}}$ \\
$\Xi^{0} \rightarrow \Sigma^{+} \ell^{-} \bar{\nu}_{\ell}$ & $1.22 \pm 0.05$ & $2.0 \pm 0.9$ \\
$\Lambda^{0} \rightarrow p \ell^{-} \bar{\nu}_{\ell}$ & $0.718 \pm 0.015$ & $1.066^{\mathrm{b}}$ \\
$\Sigma^{0} \rightarrow p \ell^{-} \bar{\nu}_{\ell}$ & $-0.340 \pm 0.017^{\mathrm{a}}$ & $-1.292^{\mathrm{b}}$ \\
$\Sigma^{-} \rightarrow n \ell^{-} \bar{\nu}_{\ell}$ & $-0.340 \pm 0.017$ & $-0.97 \pm 0.14$ \\
$\Sigma^{-} \rightarrow \Sigma^{0} e^{-} \bar{\nu}_{e}$ & $\frac{1}{2}[(1.2724 \pm 0.0023)+(-0.340 \pm 0.017)]^{\mathrm{a}}$ & $0.534^{\mathrm{b}}$ \\
$\Sigma^{-} \rightarrow \Lambda^{0} e^{-} \bar{\nu}_{e}$ & $(-0.01 \pm 0.10)^{-1}$ & $1.490^{\mathrm{b}}$ \\
$\Sigma^{0} \rightarrow \Sigma^{+} e^{-} \bar{\nu}_{e}$ & $-\frac{1}{2}[(1.2724 \pm 0.0023)+(-0.340 \pm 0.017)]^{\mathrm{a}}$ & $0.531^{\mathrm{b}}$ \\
$\Sigma^{+} \rightarrow \Lambda^{0} e^{+} \nu_{e}$ & $(-0.01 \pm 0.10)^{-1 \mathrm{a}}$ & $1.490^{\mathrm{b}}$ \\
$\Xi^{-} \rightarrow \Xi^{0} e^{-} \bar{\nu}_{e}$ & $-0.340 \pm 0.017^{\mathrm{a}}$ & $-1.432^{\mathrm{b}}$ \\
$n \rightarrow p e^{-} \bar{\nu}_{e}$ & $1.2724 \pm 0.0023$ & $1.855^{\mathrm{b}}$ \\
\hline \hline
\end{tabular}

${ }^{a}$ Values were obtained from the SU(3) flavor parametrization $F$ and $D$ given in Refs. [44,56] and the measured form factor ratios in Ref. [1].

${ }^{\mathrm{b}}$ Values were taken from Cabibbo theory [56].

Eq. (7) will be used to obtain more precise predictions in the following $S_{2}$ case.

Then the differential branching ratios of $T_{8 A} \rightarrow T_{8 B} \ell^{-} \bar{\nu}_{\ell}$ decays can be written using the helicity amplitudes

$$
\begin{aligned}
\frac{d \mathcal{B}\left(T_{8 A} \rightarrow T_{8 B} \ell^{-} \bar{\nu}_{\ell}\right)}{d q^{2}}= & \frac{G_{F}^{2}\left|V_{u q_{n}}\right|^{2} \tau_{A}\left|\vec{p}_{B}\right| q^{2}}{192 \pi^{3} m_{A}^{2}}\left(1-\frac{m_{\ell}^{2}}{q^{2}}\right)^{2} \\
& \times\left[B_{1}+\frac{m_{\ell}^{2}}{2 q^{2}} B_{2}\right]
\end{aligned}
$$

with

$$
\begin{aligned}
B_{1}= & \left|H_{\frac{1}{2} 0}\right|^{2}+\left|H_{-\frac{1}{2} 0}\right|^{2}+\left|H_{\frac{1}{2} 1}^{2}\right|^{2}+\left|H_{-\frac{1}{2}-1}\right|^{2}, \\
B_{2}= & \left|H_{\frac{1}{2} 0}\right|^{2}+\left|H_{-\frac{1}{2} 0}\right|^{2}+\left|H_{\frac{1}{2} 1}\right|^{2}+\left|H_{-\frac{1}{2}-1}\right|^{2} \\
& +3\left(\left|H_{\frac{1}{2} t}\right|^{2}+\mid H_{-\frac{1}{2}} t^{2}\right) .
\end{aligned}
$$

The differential longitudinal branching ratios $d \mathcal{B}^{L}\left(T_{8 A} \rightarrow\right.$ $\left.T_{8 B} \ell^{-} \bar{\nu}_{\ell}\right) / d q^{2}$ can be obtained from $d \mathcal{B}\left(T_{8 A} \rightarrow T_{8 B} \ell^{-} \bar{\nu}_{\ell}\right) /$ $d q^{2}$ by setting $\left|H_{\frac{1}{2}}^{2}\right|^{2}=\left|H_{-\frac{1}{2}-1}\right|^{2}=0$ in Eqs. (9) and (10).

\section{Input parameters}

The theoretical input parameters and the experimental data within the $1 \sigma$ error from Particle Data Group [1] will be used in our numerical results. And two cases will be considered in our analysis:

$S_{1}$. - In the limit of $m_{\ell}=0$, we neglect the $B_{2}$ term in Eq. (9) as in Ref. [37] and treat SU(3) flavor parameters $\left(a_{n i}\right)_{\lambda_{B} \lambda_{W}}^{V(A)}\left(q^{2}\right)$ as constants without the $q^{2}$ dependence; i.e., $B_{1}$ in Eq. (9) is constant. For simplification, the helicity information of $\lambda_{B}$ and $\lambda_{W}$ in $B_{1}$ is ignored in this case. Then there are three parameters:
$A_{31}, A_{32} e^{i \delta_{A_{32}}}$ for the $s \rightarrow u \ell^{-} \bar{\nu}_{\ell}$ transition,

$A_{21}, \bar{A}_{22} e^{i \delta_{\bar{A}_{22}}} \quad$ for the $d \rightarrow u e^{-} \bar{\nu}_{e}$ transition.

Note that $A_{31}, A_{32}, A_{21}$, and $\bar{A}_{22}$ could be complex, and we set $A_{31}\left(A_{21}\right)$ as real and add relative phase $\delta_{A_{32}}\left(\delta_{\bar{A}_{22}}\right)$ associated with $A_{32}\left(\bar{A}_{22}\right)$ in this work. From the amplitude relations between different decay modes given in the last column of Table I, we take $B_{1}=$ $A_{31}^{2}$ for the $\Xi^{-} \rightarrow \Sigma^{0} \ell^{-} \bar{\nu}_{\ell}$ decays, $B_{1}=\mid A_{31}+$ $\left.2 A_{32} e^{i \delta_{A_{32}}}\right|^{2}$ for the $\Xi^{-} \rightarrow \Lambda^{0} \ell^{-} \bar{\nu}_{\ell}$ decays, and $B_{1}=$ $\mid 2 A_{31}+A_{32} e^{\left.i \delta_{A_{32}}\right|^{2}}$ for the $\Lambda^{0} \rightarrow p \ell^{-} \bar{\nu}_{\ell}$ decays; $B_{1}$ for the other decays is similar.

$S_{2}$. - In order to obtain more precise predictions, we use the helicity amplitudes in Eq. (7). The form factors for the hyperon semileptonic decays are calculated in various approaches - for example, quark and soliton models, the $1 / N_{c}$ expansion of QCD, lattice QCD, chiral perturbation theory, etc., [46,49-59]. In this case, we choose the dipole behavior for the form factors as $[44,56]$

$$
F_{i}\left(q^{2}\right)=\frac{F_{i}(0)}{\left(1-q^{2} / M^{2}\right)^{2}},
$$

where $M=0.97(1.25) \mathrm{GeV}$ for the vector (axial vector) form factors $f_{i}\left(g_{i}\right)$ in the $s \rightarrow u \ell^{-} \bar{\nu}_{\ell}$ decays, and $M=0.84 \pm 0.04(1.08 \pm 0.08) \mathrm{GeV}$ for $f_{i}\left(g_{i}\right)$ in the $d \rightarrow u e^{-} \bar{\nu}_{e}$ decays. For the form factor ratios $g_{1}(0) / f_{1}(0)$ and $f_{2}(0) / f_{1}(0)$, they are preferentially taken from experimental measurements. If no relevant experimental measurements are available, they will be taken from Cabibbo theory [56]. The form factor ratios in Table II will be used in our results. As a result, the branching ratios depend only on the form factor $f_{1}(0)$ and the CKM matrix elemant $V_{u q_{n}}$. Then these three parameters become 
TABLE III. The experimental data and the SM predictions with the $\pm 1 \sigma$ error bar of branching ratios of $T_{8 A} \rightarrow T_{8 B} \ell \nu_{\ell}$.

\begin{tabular}{|c|c|c|c|c|}
\hline Observables & Experimental Data [1] & $\mathcal{B} r-S_{1}$ & $\mathcal{B} r-S_{2}$ & $\mathcal{B} r^{L}-S_{2}$ \\
\hline $\mathcal{B}\left(\Xi^{-} \rightarrow \Sigma^{0} e^{-} \bar{\nu}_{e}\right)\left(\times 10^{-5}\right)$ & $8.7 \pm 1.7$ & $8.12 \pm 0.60$ & $8.27 \pm 0.58$ & $5.23 \pm 0.35$ \\
\hline $\mathcal{B}\left(\Xi^{-} \rightarrow \Lambda^{0} e^{-} \bar{\nu}_{e}\right)\left(\times 10^{-4}\right)$ & $5.63 \pm 0.31$ & $1.21 \pm 0.71$ & $5.47 \pm 0.15^{\mathrm{a}}$ & $4.94 \pm 0.14$ \\
\hline $\mathcal{B}\left(\Xi^{0} \rightarrow \Sigma^{+} e^{-} \bar{\nu}_{e}\right)\left(\times 10^{-4}\right)$ & $2.52 \pm 0.08$ & $2.52 \pm 0.08^{\mathrm{a}}$ & $2.52 \pm 0.08^{\mathrm{a}}$ & $1.60 \pm 0.06$ \\
\hline $\mathcal{B}\left(\Lambda^{0} \rightarrow p e^{-} \bar{\nu}_{e}\right)\left(\times 10^{-4}\right)$ & $8.32 \pm 0.14$ & $8.32 \pm 0.14^{\mathrm{a}}$ & $8.32 \pm 0.14^{\mathrm{a}}$ & $6.05 \pm 0.13$ \\
\hline $\mathcal{B}\left(\Sigma^{0} \rightarrow p e^{-} \bar{\nu}_{e}\right)\left(\times 10^{-13}\right)$ & $\cdots$ & $2.41 \pm 0.32$ & $2.46 \pm 0.32$ & $2.01 \pm 0.26$ \\
\hline $\mathcal{B}\left(\Sigma^{-} \rightarrow n e^{-} \bar{\nu}_{e}\right)\left(\times 10^{-3}\right)$ & $1.017 \pm 0.034$ & $1.017 \pm 0.034^{\mathrm{a}}$ & $1.013 \pm 0.030^{\mathrm{a}}$ & $0.851 \pm 0.034$ \\
\hline $\mathcal{B}\left(\Xi^{-} \rightarrow \Sigma^{0} \mu^{-} \bar{\nu}_{\mu}\right)\left(\times 10^{-6}\right)$ & $\leq 800$ & $1.08 \pm 0.09$ & $1.13 \pm 0.08$ & $0.57 \pm 0.04$ \\
\hline $\mathcal{B}\left(\Xi^{-} \rightarrow \Lambda^{0} \mu^{-} \bar{\nu}_{\mu}\right)\left(\times 10^{-4}\right)$ & $3.5_{-2.2}^{+3.5}$ & $0.33 \pm 0.19$ & $1.58 \pm 0.04$ & $1.41 \pm 0.04$ \\
\hline $\mathcal{B}\left(\Xi^{0} \rightarrow \Sigma^{+} \mu^{-} \bar{\nu}_{\mu}\right)\left(\times 10^{-6}\right)$ & $2.33 \pm 0.35$ & $2.14 \pm 0.14$ & $2.18 \pm 0.1$ & $1.09 \pm 0.08$ \\
\hline $\mathcal{B}\left(\Lambda^{0} \rightarrow p \mu^{-} \bar{\nu}_{\mu}\right)\left(\times 10^{-4}\right)$ & $1.57 \pm 0.35$ & $1.35 \pm 0.02$ & $1.40 \pm 0.02$ & $0.94 \pm 0.02$ \\
\hline $\mathcal{B}\left(\Sigma^{0} \rightarrow p \mu^{-} \bar{\nu}_{\mu}\right)\left(\times 10^{-13}\right)$ & $\cdots$ & $1.05 \pm 0.14$ & $1.13 \pm 0.15$ & $0.92 \pm 0.12$ \\
\hline $\mathcal{B}\left(\Sigma^{-} \rightarrow n \mu^{-} \bar{\nu}_{\mu}\right)\left(\times 10^{-4}\right)$ & $4.5 \pm 0.4$ & $4.53 \pm 0.15$ & $4.76 \pm 0.14^{\mathrm{a}}$ & $3.99 \pm 0.17$ \\
\hline $\mathcal{B}\left(\Sigma^{-} \rightarrow \Sigma^{0} e^{-} \bar{\nu}_{e}\right)\left(\times 10^{-10}\right)$ & $\cdots$ & $4.36 \pm 4.01^{\mathrm{b}}$ & $1.35 \pm 0.28^{\mathrm{b}}$ & $1.11 \pm 0.23^{\mathrm{b}}$ \\
\hline $\mathcal{B}\left(\Sigma^{-} \rightarrow \Lambda^{0} e^{-} \bar{\nu}_{e}\right)\left(\times 10^{-5}\right)$ & $5.73 \pm 0.27$ & $5.73 \pm 0.27^{\mathrm{a}}$ & $5.73 \pm 0.27^{\mathrm{a}}$ & $3.18 \pm 0.15$ \\
\hline $\mathcal{B}\left(\Sigma^{0} \rightarrow \Sigma^{+} e^{-} \bar{\nu}_{e}\right)\left(\times 10^{-20}\right)$ & $\ldots$ & $3.41 \pm 3.20^{\mathrm{b}}$ & $0.97 \pm 0.35^{\mathrm{b}}$ & $0.80 \pm 0.28^{\mathrm{b}}$ \\
\hline $\mathcal{B}\left(\Sigma^{+} \rightarrow \Lambda^{0} e^{+} \nu_{e}\right)\left(\times 10^{-5}\right)$ & $2.0 \pm 0.5$ & $1.88 \pm 0.11$ & $1.86 \pm 0.11$ & $1.04 \pm 0.06$ \\
\hline $\mathcal{B}\left(\Xi^{-} \rightarrow \Xi^{0} e^{-} \bar{\nu}_{e}\right)\left(\times 10^{-9}\right)$ & $\leq 2.3 \times 10^{6}$ & $2.57 \pm 2.53^{b}$ & $0.42 \pm 0.24^{\mathrm{b}}$ & $0.37 \pm 0.21^{\mathrm{b}}$ \\
\hline $\mathcal{B}\left(n \rightarrow p e^{-} \bar{\nu}_{e}\right)$ & $100 \%$ & $100 \%^{\mathrm{a}}$ & $100 \%^{\mathrm{a}}$ & $(58.38 \pm 0.03) \%$ \\
\hline
\end{tabular}

${ }^{\mathrm{a}}$ Experimental data giving the final effective constraints on the parameters.

${ }^{\mathrm{b}}$ Predictions depending on the relative phase, which is not constrained well with the present data.

$A_{31}^{\prime}, A_{32}^{\prime} e^{i \delta_{A_{32}^{\prime}}}$ for the $s \rightarrow u \ell^{-} \bar{\nu}_{\ell}$ transition,

$A_{21}^{\prime}, \bar{A}_{22}^{\prime} e^{i \delta_{\bar{A}_{22}^{\prime}}}$ for the $d \rightarrow u e^{-} \bar{\nu}_{e}$ transition,

where $A_{n i}^{\prime}$ contains $f_{1}(0)$ but without the $q^{2}$ dependence, and the parametric relations of the helicity amplitudes by $A_{n i}^{\prime}$ are similar to the ones by $A_{n i}$ listed in the last column of Table I. For example, we take $f_{1}(0)=\left|A_{31}^{\prime}\right|$ for the $\Xi^{-} \rightarrow \Sigma^{0} \ell^{-} \bar{\nu}_{\ell}$ decays, $f_{1}(0)=$ $\left|A_{31}^{\prime}+2 A_{32}^{\prime} e^{i \delta_{A_{32}^{\prime}}^{\prime}}\right|$ for the $\Xi^{-} \rightarrow \Lambda^{0} \ell^{-} \bar{\nu}_{\ell}$ decays, and $f_{1}(0)=\left|-\left(2 A_{31}^{\prime}+A_{32}^{\prime} e^{i \delta_{A_{32}^{\prime}}}\right)\right|$ for the $\Lambda^{0} \rightarrow p \ell^{-} \bar{\nu}_{\ell}$ decays.

\section{Numerical results}

The results of the $12 s \rightarrow u \ell^{-} \bar{\nu}_{\ell}$ decay modes are as follows:

(a) In the $S_{1}$ case, first, we use the experimental measurements of $\mathcal{B}\left(\Xi^{0} \rightarrow \Sigma^{+} e^{-} \bar{\nu}_{e}\right)$ and $\mathcal{B}\left(\Sigma^{-} \rightarrow n e^{-} \bar{\nu}_{e}\right)$ to obtain $A_{31}$ and $A_{32}$, second, we use the data of $\mathcal{B}\left(\Lambda^{0} \rightarrow\right.$ $p e^{-} \bar{\nu}_{e}$ ) to constrain $\delta_{A_{32}}$, which varies in the region $\left[-180^{\circ}, 180^{\circ}\right]$, and then we give the predictions of relevant branching ratios. We get $A_{31}=5.87 \pm 0.21$, $A_{32}=2.57 \pm 0.06$, and $\left|\delta_{A_{32}}\right| \leq 155.90^{\circ}$, and the predictions are listed in the second column of Table III. One can see that when the branching ratio predictions satisfy the data of $\mathcal{B}\left(\Xi^{0} \rightarrow \Sigma^{+} e^{-} \bar{\nu}_{e}\right), \mathcal{B}\left(\Sigma^{-} \rightarrow n e^{-} \bar{\nu}_{e}\right)$, and $\mathcal{B}\left(\Lambda^{0} \rightarrow p e^{-} \bar{\nu}_{e}\right)$, the predictions of $\mathcal{B}\left(\Xi^{-} \rightarrow \Lambda^{0} e^{-} \bar{\nu}_{e}\right)$ and $\mathcal{B}\left(\Xi^{-} \rightarrow \Lambda^{0} \mu^{-} \bar{\nu}_{\mu}\right)$ obviously deviate from their experimental data. (b) In the $S_{2}$ case, we consider the $q^{2}$ dependence of the form factors and use all relevant experimental data to constrain the parameters $A_{31}^{\prime}, A_{32}^{\prime}$, and $\delta_{A_{32}^{\prime}}$. We get $A_{31}^{\prime}=1.04 \pm 0.04, A_{32}^{\prime}=0.98 \pm 0.03$, and $\left|\delta_{A_{32}^{\prime}}\right| \leq 28^{\circ}$, and the branching ratio predictions are given in the third column of Table III. We can see that the experimental data of $\mathcal{B}\left(\Xi^{-} \rightarrow \Lambda^{0} e^{-} \bar{\nu}_{e}, \Xi^{0} \rightarrow \Sigma^{+} e^{-} \bar{\nu}_{e}, \Lambda^{0} \rightarrow p e^{-} \bar{\nu}_{e}\right.$, $\left.\Sigma^{-} \rightarrow n e^{-} \bar{\nu}_{e}, \Sigma^{-} \rightarrow n \mu^{-} \bar{\nu}_{\mu}\right)$ give the finally effective constraints on the relevant parameters, and the SU(3) IRA predictions in the $S_{2}$ case are quite consistent with the present data within $1 \sigma$ error. We predict that $\mathcal{B}\left(\Xi^{-} \rightarrow \Sigma^{0} \mu^{-} \bar{\nu}_{\mu}\right)$ is on the $10^{-6}$ order of magnitude, which is promised to be observed by the BESIII and $\mathrm{LHCb}$ experiments.

The results of the six $d \rightarrow u e^{-} \bar{\nu}_{e}$ decay modes are as follows:

(c) Three branching ratios, $\mathcal{B}\left(\Sigma^{-} \rightarrow \Lambda^{0} e^{-} \bar{\nu}_{e}\right), \mathcal{B}\left(\Sigma^{+} \rightarrow\right.$ $\left.\Lambda^{0} e^{+} \nu_{e}\right)$, and $\mathcal{B}\left(n \rightarrow p e^{-} \bar{\nu}_{e}\right)$, are precisely measured which can be used to constrain on $A_{21}^{(\prime)}$ and $\bar{A}_{22}^{\left({ }^{\prime}\right)}$ but not on the relative phase $\delta_{\bar{A}_{22}^{\prime \prime}}$, so we have quite large errors in the predictions of $\mathcal{B}\left(\Sigma^{-} \rightarrow \Sigma^{0} e^{-} \bar{\nu}_{e}, \Sigma^{0} \rightarrow\right.$ $\left.\Sigma^{+} e^{-} \bar{\nu}_{e}, \Xi^{-} \rightarrow \Xi^{0} e^{-} \bar{\nu}_{e}\right)$. We obtain $A_{21}=$ $4.61 \pm 0.01$ and $\bar{A}_{22}=5.85 \pm 0.16$ in the $S_{1}$ case as well as $A_{21}^{\prime}=4.50 \pm 0.02$ and $\bar{A}_{22}^{\prime}=0.36 \pm 0.36$ in the $S_{2}$ case. The predictions for $\mathcal{B}\left(\Sigma^{-} \rightarrow \Sigma^{0} e^{-} \bar{\nu}_{e}\right.$, $\left.\Sigma^{0} \rightarrow \Sigma^{+} e^{-} \bar{\nu}_{e}, \Xi^{-} \rightarrow \Xi^{0} e^{-} \bar{\nu}_{e}\right)$ in the $S_{2}$ case are obviously different than that in the $S_{1}$ case. We predict that $\mathcal{B}\left(\Sigma^{-} \rightarrow \Sigma^{0} e^{-} \bar{\nu}_{e}, \Xi^{-} \rightarrow \Xi^{0} e^{-} \bar{\nu}_{e}\right)$ are on the order of $10^{-10}$ in the $S_{2}$ case, which should be tested in the future experiments. 
The longitudinal branching ratios of the $T_{8 A} \rightarrow$ $T_{8 B} \ell^{-} \bar{\nu}_{\ell}$ decays are also predicted in the $S_{2}$ case, and they are listed in the last column of Table III. Note that the lifetime of $\Sigma^{0}$ is very small, so the relevant decay branching ratios are also very small, and the same things happen in the later $\Xi^{* 0,-}, \Sigma^{* 0,-}$, and $\Delta^{0,-}$ semileptonic decays.

\section{B. $T_{10} \rightarrow T_{8} \ell^{-} \bar{\nu}_{\ell}$ semileptonic decays}

The Feynman diagram for the $T_{10} \rightarrow T_{8} \ell^{-} \bar{\nu}_{\ell}$ decays is also shown in Fig. 1. Similar to the $T_{8 A} \rightarrow T_{8 B} \ell^{-} \bar{\nu}_{\ell}$ semileptonic decays, the SU(3) IRA helicity amplitudes $H_{\lambda_{B} \lambda_{W}}^{V(A)}$ for $T_{10} \rightarrow T_{8} \ell^{-} \bar{\nu}_{\ell}$ decays can be parametrized as

$$
H_{\lambda_{B} \lambda_{W}}^{V(A), I R A}=b_{n 1} H_{n}^{k}\left(T_{10}\right)^{n i j}\left(T_{8}\right)_{[i k] j},
$$

where $b_{n 1} \equiv\left(b_{n 1}\right)_{\lambda_{B} \lambda_{W}}^{V(A)}\left(q^{2}\right)$, and where $H_{n}^{k}$ has been defined below Eq. (5). The helicity amplitudes $H_{\lambda_{B} \lambda_{W}}^{V(A)}$ for different $T_{10} \rightarrow T_{8} \ell^{-} \bar{\nu}_{\ell}$ decays are given in Table IV. And the differential branching ratios of $T_{10 A} \rightarrow T_{8 B} \ell^{-} \bar{\nu}_{\ell}$ decays can be written as

$$
\begin{aligned}
& \frac{d \mathcal{B}\left(T_{10 A} \rightarrow T_{8 B} \ell^{-} \bar{\nu}_{\ell}\right)}{d q^{2}} \\
& =\frac{G_{F}^{2}\left|V_{u q_{n}}\right|^{2} \tau_{A}\left|\vec{p}_{B}\right| q^{2}}{384 \pi^{3} m_{A}^{2}}\left(1-\frac{m_{\ell}^{2}}{q^{2}}\right)^{2}\left[B_{1}^{\prime}+\frac{m_{\ell}^{2}}{2 q^{2}} B_{2}^{\prime}\right],
\end{aligned}
$$

with

TABLE IV. The helicity amplitudes $H_{\lambda_{B} \lambda_{W}}^{V(A)}$ of the $T_{10} \rightarrow$ $T_{8} \ell^{-} \bar{\nu}_{\ell}$ decays.

\begin{tabular}{lc}
\hline \hline$H_{\lambda_{B} \lambda_{W}}^{V(A)}$ & SU(3) IRA amplitudes \\
\hline$s \rightarrow \boldsymbol{u} \ell^{-} \overline{\boldsymbol{\nu}}_{\ell}:$ & \\
$H\left(\Omega^{-} \rightarrow \Xi^{0} \ell^{-} \bar{\nu}_{\ell}\right)$ & $b_{31}$ \\
$3 \sqrt{2} H\left(\Xi^{*-} \rightarrow \Lambda^{0} \ell^{-} \bar{\nu}_{\ell}\right)$ & $b_{31}$ \\
$\sqrt{6} H\left(\Xi^{*-} \rightarrow \Sigma^{0} \ell^{-} \bar{\nu}_{\ell}\right)$ & $b_{31}$ \\
$\sqrt{3} H\left(\Xi^{* 0} \rightarrow \Sigma^{+} \ell^{-} \bar{\nu}_{\ell}\right)$ & $-b_{31}$ \\
$\sqrt{3} H\left(\Sigma^{*-} \rightarrow n \ell^{-} \bar{\nu}_{\ell}\right)$ & $-b_{31}$ \\
$\sqrt{6} H\left(\Sigma^{* 0} \rightarrow p \ell^{-} \bar{\nu}_{\ell}\right)$ & \\
$d \rightarrow u e^{-} \bar{\nu}_{e}:$ & $b_{21}$ \\
$\sqrt{3} H\left(\Xi^{*-} \rightarrow \Xi^{0} e^{-} \bar{\nu}_{e}\right)$ & $3 b_{21}$ \\
$2 \sqrt{3} H\left(\Sigma^{*-} \rightarrow \Lambda^{0} e^{-} \bar{\nu}_{e}\right)$ & $b_{21}$ \\
$\sqrt{6} H\left(\Sigma^{*-} \rightarrow \Sigma^{0} e^{-} \bar{\nu}_{e}\right)$ & $b_{21}$ \\
$\sqrt{6} H\left(\Sigma^{* 0} \rightarrow \Sigma^{+} e^{+} \nu_{e}\right)$ & $-b_{21}$ \\
$H\left(\Delta^{-} \rightarrow n e^{-} \bar{\nu}_{e}\right)$ & $-b_{21}$ \\
$\sqrt{3} H\left(\Delta^{0} \rightarrow p e^{-} \bar{\nu}_{e}\right)$ & \\
\hline \hline
\end{tabular}

$$
\begin{aligned}
B_{1}^{\prime}= & \left|H_{\frac{1}{2} 0}\right|^{2}+\left|H_{-\frac{1}{2}}\right|^{2}+\left|H_{\frac{1}{2} 1}\right|^{2}+\left|H_{-\frac{1}{2}}\right|^{2} \\
& +\left|H_{-\frac{1}{2}-1}\right|^{2}+\left|H_{\frac{1}{2}-1}\right|^{2}, \\
B_{2}^{\prime}= & \left|H_{\frac{1}{2} 0}\right|^{2}+\left|H_{-\frac{1}{2} 0}\right|^{2}+\left|H_{\frac{1}{2} 1}\right|^{2}+\left|H_{-\frac{1}{2}}\right|^{2} \\
& +\left|H_{-\frac{1}{2}-1}\right|^{2}+\left|H_{\frac{1}{2}-1}\right|^{2}+3\left(\left|H_{\frac{1}{2}}\right|^{2}+\left|H_{-\frac{1}{2} t}\right|^{2}\right) .
\end{aligned}
$$

The $S_{1}$ case given in Sec. II A will be considered in the $T_{10} \rightarrow T_{8} \ell^{-} \bar{\nu}_{\ell}$ semileptonic decays, where the $\mathrm{SU}(3)_{f}$ parameters $\left(b_{n 1}\right)_{\lambda_{B} \lambda_{W}}^{V(A)}\left(q^{2}\right)$ are treated as constant without $q^{2}$ dependence. The only parameters are $b_{31}$ for the $s \rightarrow$ $u \ell^{-} \bar{\nu}_{\ell}$ transition and $b_{21}$ for the $d \rightarrow u e^{-} \bar{\nu}_{e}$ transition, respectively.

For the $s \rightarrow u \ell^{-} \bar{\nu}_{\ell}$ transition, only $\mathcal{B}\left(\Omega^{-} \rightarrow \Xi^{0} e^{-} \bar{\nu}_{e}\right)$ has been measured. The experimental datum is listed in the second column of Table V. We use $\mathcal{B}\left(\Omega^{-} \rightarrow \Xi^{0} e^{-} \bar{\nu}_{e}\right)$ to constrain $b_{31}$, then give the predictions for other relevant decay branching ratios. The results are given in the third column of Table V. We obtain $\mathcal{B}\left(\Omega^{-} \rightarrow \Xi^{0} \mu^{-} \bar{\nu}_{\mu}\right)=$ $(3.7 \pm 1.8) \times 10^{-3}$, which promises to be measured by BESIII and LHCb. For the $d \rightarrow u e^{-} \bar{\nu}_{e}$ transition, no decay mode has been measured yet. We use $H\left(\Omega^{-} \rightarrow \Xi^{0} e^{-} \bar{\nu}_{e}\right)=$ $H\left(\Delta^{-} \rightarrow n e^{-} \bar{\nu}_{e}\right)$ by the U-spin symmetry, i.e., $b_{21}=-b_{31}$, to predict the branching ratios of the $d \rightarrow u e^{-} \bar{\nu}_{e}$ transition, which are listed in the third column of Table V, too. In Table $\mathrm{V}$, all branching ratios except for $\mathcal{B}\left(\Omega^{-} \rightarrow\right.$ $\left.\Xi^{0} e^{-} \bar{\nu}_{e}, \Xi^{0} \mu^{-} \bar{\nu}_{\mu}\right)$ are in the range of $10^{-16}$ to $10^{-15}$ since the lifetimes of the $\Xi^{* 0,-}, \Sigma^{* 0,-}$, and $\Delta^{0,-}$ baryons are very small.

TABLE V. The experimental data and the SU(3) IRA predictions with the $\pm 1 \sigma$ error bar of $\mathcal{B}\left(T_{10} \rightarrow T_{8} \ell^{-} \bar{\nu}_{\ell}\right)$.

\begin{tabular}{lcc}
\hline \hline Observables & Experimental Data[1] & $S_{1}$ \\
\hline $\mathcal{B}\left(\Omega^{-} \rightarrow \Xi^{0} e^{-} \bar{\nu}_{e}\right)\left(\times 10^{-3}\right)$ & $5.6 \pm 2.8$ & $5.6 \pm 2.8^{\mathrm{a}}$ \\
$\mathcal{B}\left(\Xi^{*-} \rightarrow \Lambda^{0} e^{-} \bar{\nu}_{e}\right)\left(\times 10^{-15}\right)$ & $\ldots$ & $6.6 \pm 4.1$ \\
$\mathcal{B}\left(\Xi^{*-} \rightarrow \Sigma^{0} e^{-} \bar{\nu}_{e}\right)\left(\times 10^{-15}\right)$ & $\ldots$ & $2.2 \pm 1.4$ \\
$\mathcal{B}\left(\Xi^{* 0} \rightarrow \Sigma^{+} e^{-} \bar{\nu}_{e}\right)\left(\times 10^{-15}\right)$ & $\ldots$ & $1.6 \pm 0.9$ \\
$\mathcal{B}\left(\Sigma^{*-} \rightarrow n e^{-} \bar{\nu}_{e}\right)\left(\times 10^{-15}\right)$ & $\ldots$ & $1.6 \pm 0.9$ \\
$\mathcal{B}\left(\Sigma^{* 0} \rightarrow p e^{-} \bar{\nu}_{e}\right)\left(\times 10^{-16}\right)$ & $\ldots$ & $9.3 \pm 5.5$ \\
$\mathcal{B}\left(\Omega^{-} \rightarrow \Xi^{0} \mu^{-} \bar{\nu}_{\mu}\right)\left(\times 10^{-3}\right)$ & $\ldots$ & $3.7 \pm 1.8$ \\
$\mathcal{B}\left(\Xi^{*-} \rightarrow \Lambda^{0} \mu^{-} \bar{\nu}_{\mu}\right)\left(\times 10^{-15}\right)$ & $\ldots$ & $4.9 \pm 3.0$ \\
$\mathcal{B}\left(\Xi^{*-} \rightarrow \Sigma^{0} \mu^{-} \bar{\nu}_{\mu}\right)\left(\times 10^{-15}\right)$ & $\ldots$ & $1.6 \pm 1.0$ \\
$\mathcal{B}\left(\Xi^{* 0} \rightarrow \Sigma^{+} \mu^{-} \bar{\nu}_{\mu}\right)\left(\times 10^{-15}\right)$ & $\ldots$ & $1.0 \pm 0.5$ \\
$\mathcal{B}\left(\Sigma^{*-} \rightarrow n \mu^{-} \bar{\nu}_{\mu}\right)\left(\times 10^{-15}\right)$ & $\ldots$ & $1.2 \pm 0.7$ \\
$\mathcal{B}\left(\Sigma^{* 0} \rightarrow p \mu^{-} \bar{\nu}_{\mu}\right)\left(\times 10^{-16}\right)$ & $\ldots$ & $7.1 \pm 4.2$ \\
$\mathcal{B}\left(\Xi^{*-} \rightarrow \Xi^{0} e^{-} \bar{\nu}_{e}\right)\left(\times 10^{-15}\right)$ & $\ldots$ & $3.6 \pm 2.2$ \\
$\mathcal{B}\left(\Sigma^{*-} \rightarrow \Lambda^{0} e^{-} \bar{\nu}_{e}\right)\left(\times 10^{-15}\right)$ & $\ldots$ & $6.2 \pm 3.3$ \\
$\mathcal{B}\left(\Sigma^{*-} \rightarrow \Sigma^{0} e^{-} \bar{\nu}_{e}\right)\left(\times 10^{-16}\right)$ & $\ldots$ & $2.7 \pm 1.4$ \\
$\mathcal{B}\left(\Sigma^{* 0} \rightarrow \Sigma^{+} e^{-} \bar{\nu}_{e}\right)\left(\times 10^{-16}\right)$ & $\ldots$ & $3.1 \pm 1.8$ \\
$\mathcal{B}\left(\Delta^{-} \rightarrow n e^{-} \bar{\nu}_{e}\right)\left(\times 10^{-15}\right)$ & $\ldots$ & $4.9 \pm 2.6$ \\
$\mathcal{B}\left(\Delta^{0} \rightarrow p e^{-} \bar{\nu}_{e}\right)\left(\times 10^{-15}\right)$ & $\ldots$ & $1.7 \pm 0.9$ \\
\hline \hline
\end{tabular}

${ }^{a}$ Experimental data giving the final effective constraints on the parameters. 


\section{NONLEPTONIC TWO-BODY DECAYS OF LIGHT BARYONS}

In this section, we discuss the two-body nonleptonic decays of the light baryons $T_{8,10} \rightarrow T_{8} M_{8}$, where $M_{8}$ are light pseudoscalar $P$ and vector $V$ meson octets under the $\mathrm{SU}(3)$ flavor symmetry of $u, d, s$ quarks:

$$
\begin{gathered}
P_{8}=\left(\begin{array}{ccc}
\frac{\eta_{8}}{\sqrt{6}}+\frac{\pi^{0}}{\sqrt{2}} & \pi^{+} & K^{+} \\
\pi^{-} & \frac{\eta_{8}}{\sqrt{6}}-\frac{\pi^{0}}{\sqrt{2}} & K^{0} \\
K^{-} & \bar{K}^{0} & -\sqrt{\frac{2}{3}} \eta_{8}
\end{array}\right), \\
V_{8}=\left(\begin{array}{ccc}
\frac{\omega_{8}}{\sqrt{6}}+\frac{\rho^{0}}{\sqrt{2}} & \rho^{+} & K^{*+} \\
\rho^{-} & \frac{\omega_{8}}{\sqrt{6}}-\frac{\rho^{0}}{\sqrt{2}} & K^{* 0} \\
K^{*-} & \bar{K}^{* 0} & -\sqrt{\frac{2}{3}} \omega_{8}
\end{array}\right) .
\end{gathered}
$$

\section{A. Weak decays of light baryons}

In the SM, as shown in Fig. 2, there are two kinds of diagrams for the nonleptonic $s$ quark decays, the tree-level diagram in Fig. 2(a) and the penguin diagram in Fig. 2(b). The effective Hamiltonian for the nonleptonic $s$ quark decays at scales $\mu<m_{c}$ can be written as [60]

$\mathcal{H}_{\text {eff }}=\frac{G_{F}}{\sqrt{2}} V_{u d} V_{u s}^{*} \sum_{i=1}^{10}\left[z_{i}(\mu)-\frac{V_{t d} V_{t s}^{*}}{V_{u d} V_{u s}^{*}} y_{i}(\mu)\right] Q_{i}(\mu)$,

where $V_{u q}$ is the CKM matrix element, and $z_{i}(\mu)$ and $y_{i}(\mu)$ are the Wilson coefficients. The four-quark operators $Q_{i}$ are

$$
\begin{aligned}
Q_{1} & =\left(\bar{d}_{\alpha} u_{\beta}\right)_{V-A}\left(\bar{u}_{\beta} s_{\alpha}\right)_{V-A}, \\
Q_{2} & =(\bar{d} u)_{V-A}(\bar{u} s)_{V-A}, \\
Q_{3,5} & =(\bar{d} s)_{V-A} \sum_{q=u, d, s}(\bar{q} q)_{V \mp A}, \\
Q_{4,6} & =\left(\bar{d}_{\beta} s_{\alpha}\right)_{V-A} \sum_{q=u, d, s}\left(\bar{q}_{\alpha} q_{\beta}\right)_{V \mp A}, \\
Q_{7,9} & =\frac{3}{2}(\bar{d} s)_{V-A} \sum_{q=u, d, s} e_{q}(\bar{q} q)_{V \pm A}, \\
Q_{8,10} & =\frac{3}{2}\left(\bar{d}_{\beta} s_{\alpha}\right)_{V-A} \sum_{q=u, d, s} e_{q}\left(\bar{q}_{\alpha} q_{\beta}\right)_{V \pm A},
\end{aligned}
$$

where $Q_{1,2}$ are current-current operators corresponding to Fig. 2(a), $Q_{3-6}\left(Q_{7-10}\right)$ are QCD (electroweak) penguin operators corresponding to Fig. 2(b). In Eq. (19), the magnetic penguin operators are ignored since their contributions are small. $C_{i}(\mu) \equiv z_{i}(\mu)-\frac{V_{t d} V_{t s}^{*}}{V_{u d} V_{u s}^{*}} y_{i}(\mu)$ at $\mu=$ $1 \mathrm{GeV}$ on $\Lambda_{\overline{M S}}^{(4)}$ in the naive dimensional regularization scheme are [60]

$$
\begin{aligned}
C_{1} & =-0.625, & C_{2}=1.361, & C_{3}=0.023, \\
C_{4} & =-0.058, & C_{5}=0.009, & C_{6}=-0.059, \\
C_{7} / \alpha_{e} & =0.021, & C_{8} / \alpha_{e}=0.027, & \\
C_{9} / \alpha_{e} & =0.036, & C_{10} / \alpha_{e}=-0.015 . &
\end{aligned}
$$

Compared with tree-level contributions related to $C_{1,2}$, the penguin contributions are suppressed by smaller Wilson coefficients $C_{3, \ldots, 10}$ and can be ignored in these decays.

The four-quark operators $Q_{i}$ can be rewritten as $\left(\bar{q}_{i} q^{k}\right)\left(\bar{q}_{j} s\right)$, with $q_{i}=(u, d)$ given as the doublet of 2 under $\mathrm{SU}(2)$ symmetry by omitting the LorentzDirac structure. Since $\left(\bar{q}_{i} q^{k}\right)\left(\bar{q}_{j} s\right)$ can be decomposed as the irreducible representations (IR) of $(\overline{2} \otimes 2 \otimes \overline{2}) s=$ $\left(\overline{2}_{p} \oplus \overline{2}_{t} \oplus 4\right) s$, one may obtain

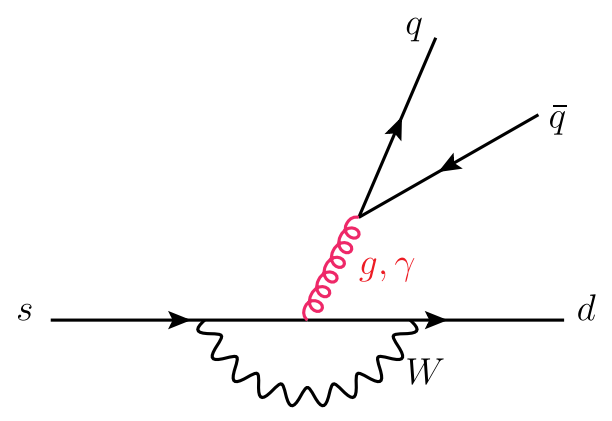

(b)

(a)

FIG. 2. Feynman diagrams for the $s$ quark decays in the SM. 
$\mathcal{O}\left(\overline{2}_{p}\right)_{2}=(\bar{u} u)(\bar{d} s)+(\bar{d} d)(\bar{d} s)$,

$\mathcal{O}\left(\overline{2}_{t}\right)_{2}=(\bar{d} u)(\bar{u} s)+(\bar{d} d)(\bar{d} s)$,

$\mathcal{O}(4)_{12}^{1}=\frac{1}{3}(\bar{u} u)(\bar{d} s)-\frac{1}{3}(\bar{d} d)(\bar{d} s)+\frac{1}{3}(\bar{d} u)(\bar{u} s)$,

and we have the relation $\mathcal{O}(4)_{21}^{1}=-\mathcal{O}(4)_{22}^{2}=\mathcal{O}(4)_{12}^{1}$ using the traceless condition. Then $Q_{1,2}, Q_{3-6}$, and $Q_{7,10}$ can be transformed under SU(2) symmetry as $\overline{2}_{p} \oplus \overline{2}_{t} \oplus 4$, $\overline{2}_{p} / \overline{2}_{t}$ and $\overline{2}_{p} \oplus \overline{2}_{t} \oplus 4$, respectively,

$$
\begin{aligned}
Q_{1} & =\mathcal{O}(4)_{12}^{1}+\frac{2}{3} \mathcal{O}\left(\overline{2}_{p}\right)_{2}-\frac{1}{3} \mathcal{O}\left(\overline{2}_{t}\right)_{2}, \\
Q_{2} & =\mathcal{O}(4)_{12}^{1}-\frac{1}{3} \mathcal{O}\left(\overline{2}_{p}\right)_{2}+\frac{2}{3} \mathcal{O}\left(\overline{2}_{t}\right)_{2}, \\
Q_{3} & =\mathcal{O}\left(\overline{2}_{p}\right)_{2}, \quad Q_{4}=\mathcal{O}\left(\overline{2}_{t}\right)_{2}, \\
Q_{5} & =\mathcal{O}^{\prime}\left(\overline{2}_{p}\right)_{2}, \quad Q_{6}=\mathcal{O}^{\prime}\left(\overline{2}_{t}\right)_{2}, \\
Q_{7} & =\frac{3}{2} \mathcal{O}^{\prime}(4)_{12}^{1}+\frac{1}{2} \mathcal{O}^{\prime}\left(\overline{2}_{p}\right)_{2}-\frac{1}{2} \mathcal{O}^{\prime}\left(\overline{2}_{t}\right)_{2}, \\
Q_{8} & =\frac{3}{2} \mathcal{O}^{\prime}(4)_{12}^{1}-\frac{1}{2} \mathcal{O}^{\prime}\left(\overline{2}_{p}\right)_{2}+\frac{1}{2} \mathcal{O}^{\prime}\left(\overline{2}_{t}\right)_{2}, \\
Q_{9} & =\frac{3}{2} \mathcal{O}(4)_{12}^{1}+\frac{1}{2} \mathcal{O}\left(\overline{2}_{p}\right)_{2}-\frac{1}{2} \mathcal{O}\left(\overline{2}_{t}\right)_{2}, \\
Q_{10} & =\frac{3}{2} \mathcal{O}(4)_{12}^{1}-\frac{1}{2} \mathcal{O}\left(\overline{2}_{p}\right)_{2}+\frac{1}{2} \mathcal{O}\left(\overline{2}_{t}\right)_{2},
\end{aligned}
$$

where $\mathcal{O}^{\prime}\left(\overline{2}_{p}\right)_{2}, \mathcal{O}^{\prime}\left(\overline{2}_{t}\right)_{2}$, and $\mathcal{O}^{\prime}(4)_{12}^{1}$ are operators related to $Q_{5,6,7,8}$, which have the same $\mathrm{SU}(3)$ structure as $\mathcal{O}\left(\overline{2}_{p}\right)_{2}, \mathcal{O}\left(\overline{2}_{t}\right)_{2}$, and $\mathcal{O}(4)_{12}^{1}$ but different Lorentz-Dirac structures.

By using the bases of the SU(2) symmetry, the effective Hamiltonian in Eq. (19) can be transformed as

$$
\begin{aligned}
\mathcal{H}_{\mathrm{eff}}^{\mathrm{IR}}= & \frac{G_{F}}{\sqrt{2}} V_{u d} V_{u s}^{*}\left[\bar{C}_{4} \mathcal{O}(4)_{12}^{1}+\bar{C}_{\overline{2}_{p}} \mathcal{O}\left(\overline{2}_{p}\right)_{2}+\bar{C}_{\overline{2}_{t}} \mathcal{O}\left(\overline{2}_{t}\right)_{2}\right. \\
& \left.+\bar{C}_{4}^{\prime} \mathcal{O}^{\prime}(4)_{12}^{1}+\bar{C}_{\overline{2}_{p}}^{\prime} \mathcal{O}^{\prime}\left(\overline{2}_{p}\right)_{2}+\bar{C}_{\overline{2}_{t}}^{\prime} \mathcal{O}^{\prime}\left(\overline{2}_{t}\right)_{2}\right]
\end{aligned}
$$

with

$$
\begin{aligned}
\bar{C}_{4} & =C_{1}+C_{2}+\frac{3}{2}\left(C_{9}+C_{10}\right), \\
\bar{C}_{\overline{2}_{p}} & =\frac{2}{3} C_{1}-\frac{1}{3} C_{2}+C_{3}+\frac{1}{2}\left(C_{9}-C_{10}\right), \\
\bar{C}_{\overline{2}_{t}} & =-\frac{1}{3} C_{1}+\frac{2}{3} C_{2}+C_{4}-\frac{1}{2}\left(C_{9}-C_{10}\right), \\
\bar{C}_{4}^{\prime} & =\frac{3}{2}\left(C_{7}+C_{8}\right), \\
\bar{C}_{\overline{2}_{p}}^{\prime} & =C_{5}+\frac{1}{2}\left(C_{7}-C_{8}\right), \\
\bar{C}_{\overline{2}_{t}}^{\prime} & =C_{6}-\frac{1}{2}\left(C_{7}-C_{8}\right) .
\end{aligned}
$$

From Eq. (21), one can see that the contributions from current-current operators related to $C_{1,2}$ are much larger than others related to $C_{3, \ldots, 10}$. So we will consider only current-current operator contributions in the following analysis. After neglecting $C_{3, \ldots, 10}$, the effective Hamiltonian in Eq. (24) can be rewritten as

$$
\begin{aligned}
\mathcal{H}_{\mathrm{eff}}^{\mathrm{IR}}= & \frac{G_{F}}{\sqrt{2}} V_{u d} V_{u s}^{*}\left\{C_{+}\left[2 H(4)+\frac{1}{3}\left(H\left(\overline{2}_{t}\right)+H\left(\overline{2}_{p}\right)\right)\right]\right. \\
& \left.+C_{-}\left(H\left(\overline{2}_{t}\right)-H\left(\overline{2}_{p}\right)\right)\right\},
\end{aligned}
$$

where $C_{ \pm} \equiv\left(C_{2} \pm C_{1}\right) / 2$, and where $H_{k}^{i j}$ is related to the $\left(\bar{q}_{i} q^{k}\right)\left(\bar{q}_{j} s\right)$ operators. From Eq. (21), one gets $C_{+}^{2} / C_{-}^{2} \approx$ $13.7 \%$, so the $C_{-}$term related to $H\left(\overline{2}_{t}\right)-H\left(\overline{2}_{p}\right)$ gives the dominant contribution to the decay branching ratios. The nonzero entries of $H_{k}^{i j}$ corresponding to current-current operators in $\mathrm{SU}(2)$ flavor space are

$H\left(\overline{2}_{p}\right)^{2}=H\left(\overline{2}_{t}\right)^{2}=1, \quad H(4)_{1}^{12}=H(4)_{1}^{21}=\frac{1}{3}$.

Note that $H(4)_{2}^{22}=-\frac{1}{3}$ contributes only to the penguin operators, and we ignore it.

In Eq. (26), the 2 irreducible representation is a linear combination of $\overline{2}_{p, t}$, so we need only consider a single $\overline{2}$ when computing amplitudes from the invariants and the reduced matrix elements [27].

The amplitudes of the $T_{8,10} \rightarrow T_{8} M_{8}$ decays can be written via the effective Hamiltonian in Eq. (19) as

$$
A\left(T_{8,10} \rightarrow T_{8} M_{8}\right)=\left\langle T_{8} M_{8}\left|\mathcal{H}_{\text {eff }}\right| T_{8,10}\right\rangle .
$$

These amplitudes may be divided into the $\mathrm{S}$ wave and $\mathrm{P}$ wave amplitudes, which have been analyzed, for instance, in heavy baryon chiral perturbation theory [61-64], and by using a relativistic chiral unitary approach based on coupled channels [65]. Moreover, since $\mathcal{H}_{\mathrm{eff}}^{\mathrm{IR}}$ is irreducible in $\mathrm{SU}(2)$ symmetry and the initial and final state baryons $\left(T_{8}, T_{10}, M_{8}\right)$ are irreducible in SU(3) symmetry, the amplitudes of $T_{8,10} \rightarrow$ $T_{8} M_{8}$ can be further written as

$$
A\left(T_{8,10} \rightarrow T_{8} M_{8}\right)=\left\langle T_{8} M_{8}\left|\mathcal{H}_{\mathrm{eff}}^{\mathrm{IR}}\right| T_{8,10}\right\rangle=A\left(\mathcal{O}_{4}\right)+A\left(\mathcal{O}_{\overline{2}}\right) .
$$

\section{1. $T_{8} \rightarrow T_{8} M_{8}$ weak decays}

Following Ref. [38], the Feynman diagrams for $T_{8} \rightarrow T_{8} M_{8}$ nonleptonic $s$ quark decays are displayed in Fig. 3, and the SU(3) IRA amplitudes are 


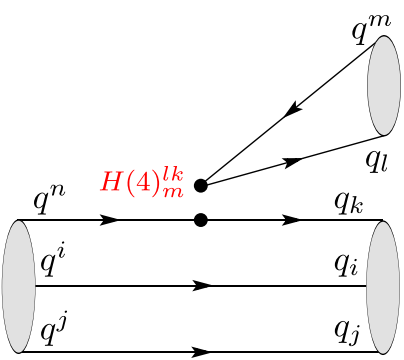

$T_{8} \rightarrow T_{8} M_{8}: a_{1, \cdots, 6}$

$T_{10} \rightarrow T_{8} M_{8}: \bar{a}_{1}$

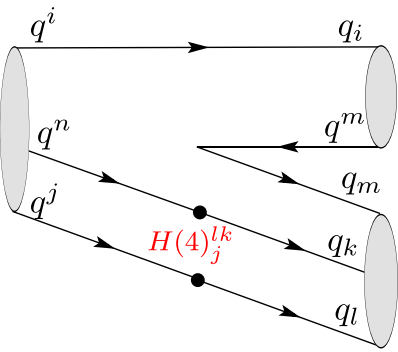

$T_{8} \rightarrow T_{8} M_{8}: d_{1, \cdots, 9}$

$T_{10} \rightarrow T_{8} M_{8}: \bar{d}_{1,2,3}$

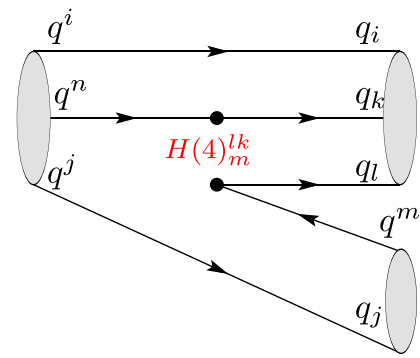

$T_{8} \rightarrow T_{8} M_{8}: b_{1, \cdots, 9}$

$T_{10} \rightarrow T_{8} M_{8}: \bar{b}_{1,2,3}$

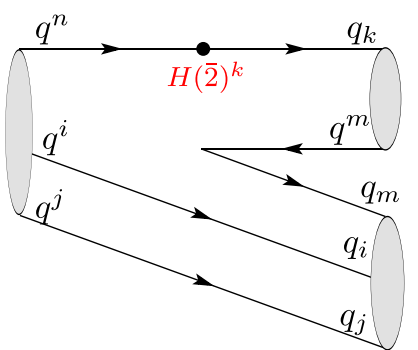

$T_{8} \rightarrow T_{8} M_{8}: e_{1, \cdots, 9}$

$T_{10} \rightarrow T_{8} M_{8}: \bar{e}_{1}$

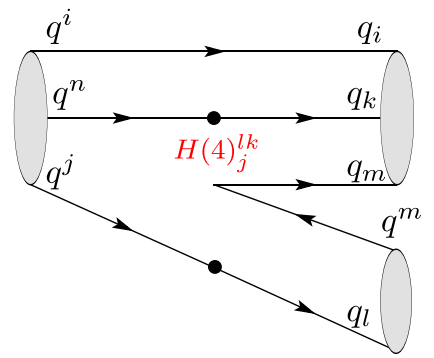

$T_{8} \rightarrow T_{8} M_{8}: c_{1, \cdots, 9}$

$T_{10} \rightarrow T_{8} M_{8}: \bar{c}_{1,2,3}$

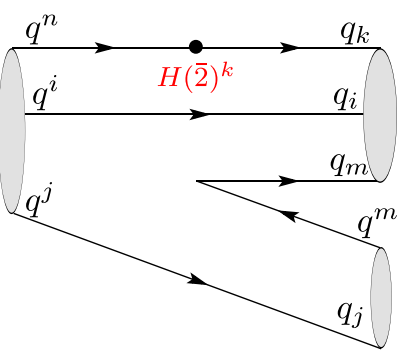

$T_{8} \rightarrow T_{8} M_{8}: f_{1, \cdots, 9}$

$T_{10} \rightarrow T_{8} M_{8}: \bar{f}_{1,2,3}$

FIG. 3. Feynman diagrams of IRA for $T_{8,10} \rightarrow T_{8} M_{8}$ nonleptonic two-body decays with $q^{n}=s$.

$$
\begin{aligned}
& A\left(T_{8} \rightarrow T_{8} M_{8}\right)^{I R A}=a_{1} H(4)_{m}^{l k}\left(T_{8}\right)^{[i j] n}\left(T_{8}\right)_{[i j] k}\left(M_{8}\right)_{l}^{m}+a_{2} H(4)_{m}^{l k}\left(T_{8}\right)^{[i j] n}\left(T_{8}\right)_{[i k] j}\left(M_{8}\right)_{l}^{m}+a_{3} H(4)_{m}^{l k}\left(T_{8}\right)^{[i j] n}\left(T_{8}\right)_{[j k] i}\left(M_{8}\right)_{l}^{m} \\
& +a_{4} H(4)_{m}^{l k}\left(T_{8}\right)^{[i n] j}\left(T_{8}\right)_{[i j] k}\left(M_{8}\right)_{l}^{m}+a_{5} H(4)_{m}^{l k}\left(T_{8}\right)^{[i n] j}\left(T_{8}\right)_{[i k] j}\left(M_{8}\right)_{l}^{m}+a_{6} H(4)_{m}^{l k}\left(T_{8}\right)^{[i n] j}\left(T_{8}\right)_{[j k] i}\left(M_{8}\right)_{l}^{m} \\
& +a_{7} H(4)_{m}^{l k}\left(T_{8}\right)^{[j n] i}\left(T_{8}\right)_{[i j] k}\left(M_{8}\right)_{l}^{m}+a_{8} H(4)_{m}^{l k}\left(T_{8}\right)^{[j n] i}\left(T_{8}\right)_{[i k] j}\left(M_{8}\right)_{l}^{m}+a_{9} H(4)_{m}^{l k}\left(T_{8}\right)^{[j n] i}\left(T_{8}\right)_{[j k] i}\left(M_{8}\right)_{l}^{m} \\
& +b_{1} H(4)_{m}^{l k}\left(T_{8}\right)^{[i j] n}\left(T_{8}\right)_{[i l] k}\left(M_{8}\right)_{j}^{m}+b_{2} H(4)_{m}^{l k}\left(T_{8}\right)^{[i j] n}\left(T_{8}\right)_{[k l] i}\left(M_{8}\right)_{j}^{m}+b_{3} H(4)_{m}^{l k}\left(T_{8}\right)^{[i j] n}\left(T_{8}\right)_{[k i] l}\left(M_{8}\right)_{j}^{m} \\
& +b_{4} H(4)_{m}^{l k}\left(T_{8}\right)^{[i n] j}\left(T_{8}\right)_{[i l] k}\left(M_{8}\right)_{j}^{m}+b_{5} H(4)_{m}^{l k}\left(T_{8}\right)^{[i n] j}\left(T_{8}\right)_{[k l] i}\left(M_{8}\right)_{j}^{m}+b_{6} H(4)_{m}^{l k}\left(T_{8}\right)^{[i n] j}\left(T_{8}\right)_{[k i] l}\left(M_{8}\right)_{j}^{m} \\
& +b_{7} H(4)_{m}^{l k}\left(T_{8}\right)^{[j n] i}\left(T_{8}\right)_{[i l] k}\left(M_{8}\right)_{j}^{m}+b_{8} H(4)_{m}^{l k}\left(T_{8}\right)^{[j n] i}\left(T_{8}\right)_{[k]] i}\left(M_{8}\right)_{j}^{m}+b_{9} H(4)_{m}^{l k}\left(T_{8}\right)^{[j n] i}\left(T_{8}\right)_{[k i] l}\left(M_{8}\right)_{j}^{m} \\
& +c_{1} H(4)_{j}^{l k}\left(T_{8}\right)^{[i j] n}\left(T_{8}\right)_{[k i] m}\left(M_{8}\right)_{l}^{m}+c_{2} H(4)_{j}^{l k}\left(T_{8}\right)^{[i j] n}\left(T_{8}\right)_{[k m] i}\left(M_{8}\right)_{l}^{m}+c_{3} H(4)_{j}^{l k}\left(T_{8}\right)^{[i j] n}\left(T_{8}\right)_{[i m] k}\left(M_{8}\right)_{l}^{m} \\
& +c_{4} H(4)_{j}^{l k}\left(T_{8}\right)^{[i n] j}\left(T_{8}\right)_{[k i] m}\left(M_{8}\right)_{l}^{m}+c_{5} H(4)_{j}^{l k}\left(T_{8}\right)^{[i n] j}\left(T_{8}\right)_{[k m] i}\left(M_{8}\right)_{l}^{m}+c_{6} H(4)_{j}^{l k}\left(T_{8}\right)^{[i n] j}\left(T_{8}\right)_{[i m] k}\left(M_{8}\right)_{l}^{m} \\
& +c_{7} H(4)_{j}^{l k}\left(T_{8}\right)^{[j n] i}\left(T_{8}\right)_{[k i] m}\left(M_{8}\right)_{l}^{m}+c_{8} H(4)_{j}^{l k}\left(T_{8}\right)^{[j n] i}\left(T_{8}\right)_{[k m] i}\left(M_{8}\right)_{l}^{m}+c_{9} H(4)_{j}^{l k}\left(T_{8}\right)^{[j n] i}\left(T_{8}\right)_{[i m] k}\left(M_{8}\right)_{l}^{m} \\
& +d_{1} H(4)_{j}^{l k}\left(T_{8}\right)^{[i j] n}\left(T_{8}\right)_{[m k] l}\left(M_{8}\right)_{i}^{m}+d_{2} H(4)_{j}^{l k}\left(T_{8}\right)^{[i j] n}\left(T_{8}\right)_{[m l] k}\left(M_{8}\right)_{i}^{m}+d_{3} H(4)_{j}^{l k}\left(T_{8}\right)^{[i j] n}\left(T_{8}\right)_{[k l] m}\left(M_{8}\right)_{i}^{m} \\
& +d_{4} H(4)_{j}^{l k}\left(T_{8}\right)^{[i n] j}\left(T_{8}\right)_{[m k] l}\left(M_{8}\right)_{i}^{m}+d_{5} H(4)_{j}^{l k}\left(T_{8}\right)^{[i n] j}\left(T_{8}\right)_{[m l] k}\left(M_{8}\right)_{i}^{m}+d_{6} H(4)_{j}^{l k}\left(T_{8}\right)^{[i n] j}\left(T_{8}\right)_{[k l] m}\left(M_{8}\right)_{i}^{m} \\
& +d_{7} H(4)_{j}^{l k}\left(T_{8}\right)^{[j n] i}\left(T_{8}\right)_{[m k] l}\left(M_{8}\right)_{i}^{m}+d_{8} H(4)_{j}^{l k}\left(T_{8}\right)^{[j n] i}\left(T_{8}\right)_{[m l] k}\left(M_{8}\right)_{i}^{m}+d_{9} H(4)_{j}^{l k}\left(T_{8}\right)^{[j n] i}\left(T_{8}\right)_{[k l] m}\left(M_{8}\right)_{i}^{m} \\
& +e_{1} H(\overline{2})^{k}\left(T_{8}\right)^{[i j] n}\left(T_{8}\right)_{[i j] m}\left(M_{8}\right)_{k}^{m}+e_{2} H(\overline{2})^{k}\left(T_{8}\right)^{[i j] n}\left(T_{8}\right)_{[i m] j}\left(M_{8}\right)_{k}^{m}+e_{3} H(\overline{2})^{k}\left(T_{8}\right)^{[i j] n}\left(T_{8}\right)_{[j m] i}\left(M_{8}\right)_{k}^{m} \\
& +e_{4} H(\overline{2})^{k}\left(T_{8}\right)^{[i n] j}\left(T_{8}\right)_{[i j] m}\left(M_{8}\right)_{k}^{m}+e_{5} H(\overline{2})^{k}\left(T_{8}\right)^{[i n] j}\left(T_{8}\right)_{[i m] j}\left(M_{8}\right)_{k}^{m}+e_{6} H(\overline{2})^{k}\left(T_{8}\right)^{[i n] j}\left(T_{8}\right)_{[j m] i}\left(M_{8}\right)_{k}^{m} \\
& +e_{7} H(\overline{2})^{k}\left(T_{8}\right)^{[j n] i}\left(T_{8}\right)_{[i j] m}\left(M_{8}\right)_{k}^{m}+e_{8} H(\overline{2})^{k}\left(T_{8}\right)^{[j n] i}\left(T_{8}\right)_{[i m] j}\left(M_{8}\right)_{k}^{m}+e_{9} H(\overline{2})^{k}\left(T_{8}\right)^{[j n] i}\left(T_{8}\right)_{[j m] i}\left(M_{8}\right)_{k}^{m} \\
& +f_{1} H(\overline{2})^{k}\left(T_{8}\right)^{[i j] n}\left(T_{8}\right)_{[k i] m}\left(M_{8}\right)_{j}^{m}+f_{2} H(\overline{2})^{k}\left(T_{8}\right)^{[i j] n}\left(T_{8}\right)_{[k m] i}\left(M_{8}\right)_{j}^{m}+f_{3} H(\overline{2})^{k}\left(T_{8}\right)^{[i j] n}\left(T_{8}\right)_{[i m] k}\left(M_{8}\right)_{j}^{m} \\
& +f_{4} H(\overline{2})^{k}\left(T_{8}\right)^{[i n] j}\left(T_{8}\right)_{[k i] m}\left(M_{8}\right)_{j}^{m}+f_{5} H(\overline{2})^{k}\left(T_{8}\right)^{[i n] j}\left(T_{8}\right)_{[k m] i}\left(M_{8}\right)_{j}^{m}+f_{6} H(\overline{2})^{k}\left(T_{8}\right)^{[i n] j}\left(T_{8}\right)_{[i m] k}\left(M_{8}\right)_{j}^{m} \\
& +f_{7} H(\overline{2})^{k}\left(T_{8}\right)^{[j n] i}\left(T_{8}\right)_{[k i] m}\left(M_{8}\right)_{j}^{m}+f_{8} H(\overline{2})^{k}\left(T_{8}\right)^{[j n] i}\left(T_{8}\right)_{[k m] i}\left(M_{8}\right)_{j}^{m}+f_{9} H(\overline{2})^{k}\left(T_{8}\right)^{[j n] i}\left(T_{8}\right)_{[i m] k}\left(M_{8}\right)_{j}^{m} \text {, }
\end{aligned}
$$


where the coefficients $a_{i}, b_{i}, c_{i}, d_{i}, e_{i}, f_{i}$ are constants which contain the Wilson coefficients, CKM matrix elements, and information about QCD dynamics. Using the fact that $H(4)_{c}^{a b}$ is symmetric in upper indices, $b_{i}$ and $d_{i}$ terms can be simplified as

$$
\begin{aligned}
& b_{3}=-b_{1}, \quad b_{6}=-b_{4}, \quad b_{9}=-b_{7}, \quad b_{2}=b_{5}=b_{8}=0 \\
& d_{2}=d_{1}, \quad d_{5}=d_{4}, \quad d_{8}=d_{7}, \quad d_{3}=d_{6}=d_{9}=0
\end{aligned}
$$

In addition, using the facts that $i, j$ are antisymmetric in $T_{8}^{[i j] n}$ and that $i, j$ indices are arbitrary in $e_{i}$ terms, we have $a_{3}=-a_{2}, \quad a_{7}=-a_{4}, \quad a_{8}=a_{6}, \quad a_{9}=a_{5}, \quad e_{3}=-e_{2}, \quad e_{7}=-e_{4}, \quad e_{8}=e_{6}, \quad e_{9}=e_{5}$

Finally, Eq. (33) can be simplified as

$$
\begin{aligned}
& A\left(T_{8} \rightarrow T_{8} M_{8}\right)^{I R A, J}=a_{1} H(4)_{m}^{l k}\left(T_{8}\right)^{[i j] n}\left(T_{8}\right)_{[i j] k}\left(M_{8}\right)_{l}^{m}+a_{2} H(4)_{m}^{l k}\left(T_{8}\right)^{[i j] n}\left(T_{8}\right)_{[i k] j}\left(M_{8}\right)_{l}^{m} \\
& +a_{4} H(4)_{m}^{l k}\left(T_{8}\right)^{[i n] j}\left(T_{8}\right)_{[i j] k}\left(M_{8}\right)_{l}^{m}+a_{5} H(4)_{m}^{l k}\left(T_{8}\right)^{[i n] j}\left(T_{8}\right)_{[i k] j}\left(M_{8}\right)_{l}^{m}+a_{6} H(4)_{m}^{l k}\left(T_{8}\right)^{[i n] j}\left(T_{8}\right)_{[j k] i}\left(M_{8}\right)_{l}^{m} \\
& +b_{1} H(4)_{m}^{l k}\left(T_{8}\right)^{[i j] n}\left(T_{8}\right)_{[i l] k}\left(M_{8}\right)_{j}^{m}+b_{4} H(4)_{m}^{l k}\left(T_{8}\right)^{[i n] j}\left(T_{8}\right)_{[i l] k}\left(M_{8}\right)_{j}^{m}+b_{7} H(4)_{m}^{l k}\left(T_{8}\right)^{[j n] i}\left(T_{8}\right)_{[i l] k}\left(M_{8}\right)_{j}^{m} \\
& +c_{1} H(4)_{j}^{l k}\left(T_{8}\right)^{[i j] n}\left(T_{8}\right)_{[k i] m}\left(M_{8}\right)_{l}^{m}+c_{2} H(4)_{j}^{l k}\left(T_{8}\right)^{[i j] n}\left(T_{8}\right)_{[k m] i}\left(M_{8}\right)_{l}^{m}+c_{3} H(4)_{j}^{l k}\left(T_{8}\right)^{[i j] n}\left(T_{8}\right)_{[i m] k}\left(M_{8}\right)_{l}^{m} \\
& +c_{4} H(4)_{j}^{l k}\left(T_{8}\right)^{[i n] j}\left(T_{8}\right)_{[k i] m}\left(M_{8}\right)_{l}^{m}+c_{5} H(4)_{j}^{l k}\left(T_{8}\right)^{[i n] j}\left(T_{8}\right)_{[k m] i}\left(M_{8}\right)_{l}^{m}+c_{6} H(4)_{j}^{l k}\left(T_{8}\right)^{[i n] j}\left(T_{8}\right)_{[i m] k}\left(M_{8}\right)_{l}^{m} \\
& +c_{7} H(4)_{j}^{l k}\left(T_{8}\right)^{[j n] i}\left(T_{8}\right)_{[k i] m}\left(M_{8}\right)_{l}^{m}+c_{8} H(4)_{j}^{l k}\left(T_{8}\right)^{[j n] i}\left(T_{8}\right)_{[k m] i}\left(M_{8}\right)_{l}^{m}+c_{9} H(4)_{j}^{l k}\left(T_{8}\right)^{[j n] i}\left(T_{8}\right)_{[i m] k}\left(M_{8}\right)_{l}^{m} \\
& +d_{1} H(4)_{j}^{l k}\left(T_{8}\right)^{[i j] n}\left(T_{8}\right)_{[m k] l}\left(M_{8}\right)_{i}^{m}+d_{4} H(4)_{j}^{l k}\left(T_{8}\right)^{[i n] j}\left(T_{8}\right)_{[m k] l}\left(M_{8}\right)_{i}^{m}+d_{7} H(4)_{j}^{l k}\left(T_{8}\right)^{[j n] i}\left(T_{8}\right)_{[m k] l}\left(M_{8}\right)_{i}^{m} \\
& +e_{1} H(\overline{2})^{k}\left(T_{8}\right)^{[i j] n}\left(T_{8}\right)_{[i j] m}\left(M_{8}\right)_{k}^{m}+e_{2} H(\overline{2})^{k}\left(T_{8}\right)^{[i j] n}\left(T_{8}\right)_{[i m] j}\left(M_{8}\right)_{k}^{m} \\
& +e_{4} H(\overline{2})^{k}\left(T_{8}\right)^{[i n] j}\left(T_{8}\right)_{[i j] m}\left(M_{8}\right)_{k}^{m}+e_{5} H(\overline{2})^{k}\left(T_{8}\right)^{[i n] j}\left(T_{8}\right)_{[i m] j}\left(M_{8}\right)_{k}^{m}+e_{6} H(\overline{2})^{k}\left(T_{8}\right)^{[i n] j}\left(T_{8}\right)_{[j m] i}\left(M_{8}\right)_{k}^{m} \\
& +f_{1} H(\overline{2})^{k}\left(T_{8}\right)^{[i j] n}\left(T_{8}\right)_{[k i] m}\left(M_{8}\right)_{j}^{m}+f_{2} H(\overline{2})^{k}\left(T_{8}\right)^{[i j] n}\left(T_{8}\right)_{[k m] i}\left(M_{8}\right)_{j}^{m}+f_{3} H(\overline{2})^{k}\left(T_{8}\right)^{[i j] n}\left(T_{8}\right)_{[i m] k}\left(M_{8}\right)_{j}^{m} \\
& +f_{4} H(\overline{2})^{k}\left(T_{8}\right)^{[i n] j}\left(T_{8}\right)_{[k i] m}\left(M_{8}\right)_{j}^{m}+f_{5} H(\overline{2})^{k}\left(T_{8}\right)^{[i n] j}\left(T_{8}\right)_{[k m] i}\left(M_{8}\right)_{j}^{m}+f_{6} H(\overline{2})^{k}\left(T_{8}\right)^{[i n] j}\left(T_{8}\right)_{[i m] k}\left(M_{8}\right)_{j}^{m} \\
& +f_{7} H(\overline{2})^{k}\left(T_{8}\right)^{[j n] i}\left(T_{8}\right)_{[k i] m}\left(M_{8}\right)_{j}^{m}+f_{8} H(\overline{2})^{k}\left(T_{8}\right)^{[j n] i}\left(T_{8}\right)_{[k m] i}\left(M_{8}\right)_{j}^{m}+f_{9} H(\overline{2})^{k}\left(T_{8}\right)^{[j n] i}\left(T_{8}\right)_{[i m] k}\left(M_{8}\right)_{j}^{m} .
\end{aligned}
$$

In Table VI, we list the IRA amplitudes of the $T_{8} \rightarrow T_{8} P_{8}$ weak decays, which include the $H(4)_{1}^{12}, H(4)_{2}^{22}$, and $H(\overline{2})^{2}$ terms. The corresponding $T_{8} \rightarrow T_{8} V_{8}$ weak decays have the same relations as the $T_{8} \rightarrow T_{8} P_{8}$ weak decays. If only considering the dominant contributions from $H(\overline{2})^{2}$ and redefining the parameters

$$
\begin{aligned}
& A_{1}=2\left(e_{5}+e_{6}\right)+\left(f_{4}+f_{5}+f_{7}+f_{8}\right), \\
& A_{2}=2\left(e_{5}+e_{6}\right)+\left(f_{5}+f_{6}+f_{8}+f_{9}\right), \\
& A_{3}=2\left(e_{5}+e_{6}\right)-\left(f_{4}-f_{5}+f_{7}-f_{8}\right), \\
& A_{4}=4\left(e_{1}+e_{2}+e_{4}\right)+2\left(e_{5}-e_{6}\right)-\left(2 f_{1}+2 f_{2}+f_{4}+f_{5}-f_{7}-f_{8}\right), \\
& A_{5}=2\left(e_{1}+e_{2}+e_{4}+2 e_{5}+e_{6}\right)-\left(f_{1}+2 f_{2}+f_{3}\right)+\left(f_{7}+2 f_{8}+f_{9}\right),
\end{aligned}
$$

the IRA amplitudes can be greatly simplified, as listed in the last column of Table VI, in which we can easily see the relations of different decay amplitudes.

The branching ratios of $T_{8} \rightarrow T_{8} P_{8}$ can be written as

$$
\mathcal{B}\left(T_{8 A} \rightarrow T_{8 B} P_{8}\right)=\frac{\tau_{A}\left|p_{c m}\right|}{8 \pi m_{A}^{2}}\left|A\left(T_{8 A} \rightarrow T_{8 B} P_{8}\right)\right|^{2}
$$

For more accurate results, we will consider the mass difference in the amplitudes [66] 
TABLE VI. The SU(3) IRA amplitudes of the $T_{8} \rightarrow T_{8} P$ weak decays.

\begin{tabular}{|c|c|c|c|c|}
\hline Amplitudes & $H(4)_{1}^{12}$ & $H(4)_{2}^{22}$ & $H(\overline{2})^{2}$ & $\begin{array}{l}\text { Simplified } \\
\text { amplitudes }\end{array}$ \\
\hline$\sqrt{2} A\left(\Sigma^{+} \rightarrow p \pi^{0}\right)$ & $\begin{array}{c}-2\left(a_{5}+a_{6}\right)-2\left(b_{4}+b_{7}\right) \\
\quad+\left(c_{4}+2 c_{5}+c_{6}\right) \\
+\left(c_{7}+2 c_{8}+c_{9}\right)-2\left(d_{4}+d_{7}\right)\end{array}$ & $2\left(a_{5}+a_{6}\right)$ & $\begin{array}{l}2\left(e_{5}+e_{6}\right)+f_{4} \\
\quad+f_{5}+f_{7}+f_{8}\end{array}$ & $A_{1}$ \\
\hline$A\left(\Sigma^{+} \rightarrow n \pi^{+}\right)$ & $\left(c_{4}-c_{6}+c_{7}-c_{9}\right)+2\left(d_{4}+d_{7}\right)$ & $-2\left(b_{4}+b_{7}\right)$ & $f_{4}-f_{6}+f_{7}-f_{9}$ & $A_{1}-A_{2}$ \\
\hline$A\left(\Sigma^{-} \rightarrow n \pi^{-}\right)$ & $-2\left(a_{5}+a_{6}\right)-2\left(b_{4}+b_{7}\right)$ & $\begin{array}{c}-\left(c_{5}+c_{6}+c_{8}+c_{9}\right) \\
+2\left(d_{4}+d_{7}\right)\end{array}$ & $\begin{array}{c}-2\left(e_{5}+e_{6}\right) \\
-\left(f_{5}+f_{6}+f_{8}+f_{9}\right) \\
\end{array}$ & $-A_{2}$ \\
\hline$\sqrt{2} A\left(\Sigma^{0} \rightarrow p \pi^{-}\right)$ & $\begin{array}{c}2\left(a_{5}+a_{6}\right)-2\left(b_{4}+b_{7}\right) \\
-\left(c_{4}-c_{6}+c_{7}-c_{9}\right)-2\left(d_{4}+d_{7}\right) \\
\end{array}$ & $c_{4}+c_{5}+c_{7}+c_{8}$ & $2\left(e_{5}+e_{6}\right)+f_{4}+f_{5}+f_{7}+f_{8}$ & $A_{1}$ \\
\hline $2 A\left(\Sigma^{0} \rightarrow n \pi^{0}\right)$ & $\begin{array}{c}-2\left(a_{5}+a_{6}\right)+2\left(b_{4}+b_{7}\right) \\
+\left(c_{4}+2 c_{5}+c_{6}\right) \\
+\left(c_{7}+2 c_{8}+c_{9}\right)-2\left(d_{4}+d_{7}\right)\end{array}$ & $\begin{array}{c}2\left(a_{5}+a_{6}\right)+2\left(b_{4}+b_{7}\right) \\
-\left(c_{4}-c_{6}+c_{7}-c_{9}\right) \\
-2\left(d_{4}+d_{7}\right)\end{array}$ & $\begin{array}{c}2\left(e_{5}+e_{6}\right) \\
-\left(f_{4}-f_{5}+f_{7}-f_{8}\right)\end{array}$ & $A_{3}$ \\
\hline$\sqrt{6} A\left(\Lambda^{0} \rightarrow p \pi^{-}\right)$ & $\begin{aligned} &-4\left(a_{1}+a_{2}+a_{4}\right)-2\left(a_{5}-a_{6}\right) \\
&-2\left(2 b_{1}+b_{4}-b_{7}\right) \\
&+\left(2 c_{1}\right.\left.-2 c_{3}+c_{4}-c_{6}-c_{7}+c_{9}\right) \\
&+2\left(2 d_{1}+d_{4}-d_{7}\right) \\
&\end{aligned}$ & $\begin{array}{l}2 c_{1}+2 c_{2}+c_{4} \\
+c_{5}-c_{7}-c_{8}\end{array}$ & $\begin{array}{l}-4\left(e_{1}+e_{2}+e_{4}\right)-2\left(e_{5}-e_{6}\right) \\
+\left(2 f_{1}+2 f_{2}+f_{4}+f_{5}-f_{7}-f_{8}\right)\end{array}$ & $-A_{4}$ \\
\hline $2 \sqrt{3} A\left(\Lambda^{0} \rightarrow n \pi^{0}\right)$ & $\begin{array}{c}-4\left(a_{1}+a_{2}+a_{4}\right)-2\left(a_{5}-a_{6}\right) \\
-2\left(2 b_{1}+b_{4}-b_{7}\right) \\
-2\left(c_{1}+2 c_{2}+c_{3}\right) \\
-\left(c_{4}+2 c_{5}+c_{6}\right)+\left(c_{7}+2 c_{8}+c_{9}\right) \\
+2\left(2 d_{1}+d_{4}-d_{7}\right)\end{array}$ & $\begin{array}{l}4\left(a_{1}+a_{2}+a_{4}\right) \\
\quad+2\left(a_{5}-a_{6}\right) \\
+2\left(2 b_{1}+b_{4}-b_{7}\right) \\
\left(-2 c_{1}+2 c_{3}-c_{4}\right. \\
\left.\quad+c_{6}+c_{7}-c_{9}\right) \\
-2\left(2 d_{1}+d_{4}-d_{7}\right) \\
\end{array}$ & $\begin{array}{c}4\left(e_{1}+e_{2}+e_{4}\right)+2\left(e_{5}-e_{6}\right) \\
-\left(2 f_{1}+2 f_{2}+f_{4}+f_{5}-f_{7}-f_{8}\right)\end{array}$ & $A_{4}$ \\
\hline$\sqrt{6} A\left(\Xi^{-} \rightarrow \Lambda^{0} \pi^{-}\right)$ & $2\left(a_{1}+a_{2}+a_{4}+2 a_{5}+a_{6}\right)$ & $\begin{array}{l}-\left(c_{1}+2 c_{2}+c_{3}\right) \\
\quad+\left(c_{7}+2 c_{8}+c_{9}\right)\end{array}$ & $\begin{array}{c}2\left(e_{1}+e_{2}+e_{4}+2 e_{5}+e_{6}\right) \\
-\left(f_{1}+2 f_{2}+f_{3}\right) \\
+\left(f_{7}+2 f_{8}+f_{9}\right)\end{array}$ & $A_{5}$ \\
\hline $2 \sqrt{3} A\left(\Xi^{0} \rightarrow \Lambda^{0} \pi^{0}\right)$ & $\begin{array}{c}2\left(a_{1}+a_{2}+a_{4}+2 a_{5}+a_{6}\right) \\
2\left(c_{1}+2 c_{2}+c_{3}\right)-2\left(c_{7}+2 c_{8}+c_{9}\right)\end{array}$ & $\begin{array}{c}-2\left(a_{1}+a_{2}+a_{4}\right. \\
\left.+2 a_{5}+a_{6}\right)\end{array}$ & $\begin{array}{c}-2\left(e_{1}+e_{2}+e_{4}+2 e_{5}+e_{6}\right) \\
+\left(f_{1}+2 f_{2}+f_{3}\right) \\
-\left(f_{7}+2 f_{8}+f_{9}\right)\end{array}$ & $-A_{5}$ \\
\hline
\end{tabular}

$$
A\left(T_{8 A} \rightarrow T_{8 B} P_{8}\right) \propto \frac{m_{A}}{m_{B}} p_{c m} N_{B} N_{A}
$$

with

$$
\begin{aligned}
p_{c m} & =\frac{1}{2 m_{A}} \sqrt{\left(m_{A}^{2}-\left(m_{B}+m_{P}\right)^{2}\right)\left(m_{A}^{2}-\left(m_{B}-m_{P}\right)^{2}\right)} \\
N_{A} & =\sqrt{2 m_{A}} \\
N_{B} & =\sqrt{\frac{\left(m_{A}+m_{B}\right)^{2}-m_{P}^{2}}{2 m_{A}}}
\end{aligned}
$$

The experimental measurements with a $\pm 1 \sigma$ error bar of the $T_{8} \rightarrow T_{8} P_{8}$ weak decays are listed in the second column of Table VII. There are four real parameters $\left(A_{1}, A_{2} e^{i \phi_{A}}, A_{3}\right)$ for five $\Sigma \rightarrow p \pi, n \pi$ decays; one can obtain $A_{1}=2.48 \pm 0.01, A_{2}=1.74 \pm 0.01$, and $\left|\phi_{A}\right| \leq$ $45.35^{\circ}$ by using the data of $\mathcal{B}\left(\Sigma^{+} \rightarrow p \pi^{0}, n \pi^{+}, \Sigma^{-} \rightarrow n \pi^{-}\right)$. Furthermore, $\mathcal{B}\left(\Sigma^{0} \rightarrow p \pi^{-}\right)$could be obtained in terms of $A_{1}$. In addition, the five $\Sigma \rightarrow n \pi, p \pi$ decay modes also have the isospin relations

$$
\begin{aligned}
& A\left(\Sigma^{+} \rightarrow p \pi^{0}\right)=-\frac{\sqrt{2}}{3}\left(A_{\frac{1}{2}}-A_{\frac{3}{2}}\right), \\
& A\left(\Sigma^{+} \rightarrow n \pi^{+}\right)=\frac{1}{3}\left(2 A_{\frac{1}{2}}+A_{\frac{3}{2}}\right), \\
& A\left(\Sigma^{-} \rightarrow n \pi^{-}\right)=-\frac{\sqrt{2}}{3}\left(A_{\frac{1}{2}}-A_{\frac{3}{2}}\right), \\
& A\left(\Sigma^{0} \rightarrow p \pi^{-}\right)=A_{\frac{3}{2}}, \\
& A\left(\Sigma^{0} \rightarrow n \pi^{0}\right)=\frac{1}{3}\left(A_{\frac{1}{2}}+2 A_{\frac{3}{2}}\right) .
\end{aligned}
$$

There are three real parameters $\left(A_{\frac{1}{2}}, A_{\frac{3}{2}} e^{i \phi_{13}}\right)$ in Eq. (38). Using the data of $\mathcal{B}\left(\Sigma^{+} \rightarrow p \pi^{0}, \Sigma^{+} \rightarrow n \pi^{+}, \Sigma^{-} \rightarrow n \pi^{-}\right)$, one can get $\mathcal{B}\left(\Sigma^{0} \rightarrow p \pi^{-}\right)$and $\mathcal{B}\left(\Sigma^{0} \rightarrow n \pi^{0}\right)$, which are listed in the last column of Table VII. We can see that SU(3) IRA and isospin relations give consistent predictions for $\mathcal{B}\left(\Sigma^{0} \rightarrow p \pi^{-}\right)$.

For $\Lambda^{0} \rightarrow p \pi^{-}, n \pi^{0}$ decays, there is only one parameter, $A_{4}$. We first get the value of $\left|A_{4}\right|$ from the data of $\mathcal{B}\left(\Lambda^{0} \rightarrow p \pi^{-}\right)$, then, further considering the experimental data of $\mathcal{B}\left(\Lambda^{0} \rightarrow n \pi^{0}\right)$, we finally give the predictions of $\mathcal{B}\left(\Lambda^{0} \rightarrow p \pi^{-}, n \pi^{0}\right)$ in the third column of Table VII. 
TABLE VII. The experimental measurements and the SM predictions with a $\pm 1 \sigma$ error bar of branching ratios of the $T_{8} \rightarrow T_{8} P_{8}$ weak decays.

\begin{tabular}{lccc}
\hline \hline Observables & Experimental Data [1] & SU(3) IRA & Isospin relations \\
\hline $\mathcal{B}\left(\Sigma^{+} \rightarrow p \pi^{0}\right)\left(\times 10^{-2}\right)$ & $51.57 \pm 0.30$ & $51.57 \pm 0.30^{\mathrm{a}}$ & $51.57 \pm 0.30^{\mathrm{a}}$ \\
$\mathcal{B}\left(\Sigma^{+} \rightarrow n \pi^{+}\right)\left(\times 10^{-2}\right)$ & $48.31 \pm 0.30$ & $48.31 \pm 0.30^{\mathrm{a}}$ & $48.31 \pm 0.30^{\mathrm{a}}$ \\
$\mathcal{B}\left(\Sigma^{-} \rightarrow n \pi^{-}\right)\left(\times 10^{-2}\right)$ & $99.848 \pm 0.005$ & $99.848 \pm 0.005^{\mathrm{a}}$ & $99.848 \pm 0.005^{\mathrm{a}}$ \\
$\mathcal{B}\left(\Sigma^{0} \rightarrow p \pi^{-}\right)\left(\times 10^{-10}\right)$ & $\ldots$ & $4.82 \pm 0.49$ & $4.82 \pm 0.50$ \\
$\mathcal{B}\left(\Sigma^{0} \rightarrow n \pi^{0}\right)\left(\times 10^{-10}\right)$ & $\cdots$ & $\cdots$ & $2.41 \pm 0.27$ \\
$\mathcal{B}\left(\Lambda^{0} \rightarrow p \pi^{-}\right)\left(\times 10^{-2}\right)$ & $63.9 \pm 0.5$ & $64.19 \pm 0.21^{\mathrm{a}}$ & $\ldots$ \\
$\mathcal{B}\left(\Lambda^{0} \rightarrow n \pi^{0}\right)\left(\times 10^{-2}\right)$ & $35.8 \pm 0.5$ & $35.42 \pm 0.12^{\mathrm{a}}$ & $\ldots$ \\
$\mathcal{B}\left(\Xi^{-} \rightarrow \Lambda^{0} \pi^{-}\right)\left(\times 10^{-2}\right)$ & $99.887 \pm 0.035$ & $99.887 \pm 0.035^{\mathrm{a}}$ & $\ldots$ \\
$\mathcal{B}\left(\Xi^{0} \rightarrow \Lambda^{0} \pi^{0}\right)\left(\times 10^{-2}\right)$ & $99.524 \pm 0.012$ & $80.016 \pm 3.746^{\mathrm{b}}$ & $\cdots$ \\
\hline \hline
\end{tabular}

${ }^{\mathrm{a}}$ Experimental data have been used to give the effective constraints of the parameters.

${ }^{\mathrm{b}}$ Experimental data have not been used to constrain the parameters.

One can see that the data of both $\mathcal{B}\left(\Lambda^{0} \rightarrow p \pi^{-}\right)$and $\mathcal{B}\left(\Lambda^{0} \rightarrow n \pi^{0}\right)$ give the effective bounds on the parameter $\left|A_{4}\right|$, and the IRA predictions for $\mathcal{B}\left(\Lambda^{0} \rightarrow p \pi^{-}, n \pi^{0}\right)$ are in agreement with the present data. Note that, if only considering the experimental constraint from $\mathcal{B}\left(\Lambda^{0} \rightarrow p \pi^{-}\right)$, the prediction of $\mathcal{B}\left(\Lambda^{0} \rightarrow p \pi^{-}\right)$given in the third column of Table VII would be the same as the experimental datum. The slight difference between the prediction and the datum comes from the experimental constraint of $\mathcal{B}\left(\Lambda^{0} \rightarrow n \pi^{0}\right)$.

For $\Xi^{-} \rightarrow \Lambda^{0} \pi^{-}$and $\Xi^{0} \rightarrow \Lambda^{0} \pi^{0}$ decays, there is only one parameter $A_{5}$. We use the data of $\mathcal{B}\left(\Xi^{-} \rightarrow \Lambda^{0} \pi^{-}\right)$to obtain
$\left|A_{5}\right|$, then predict $\mathcal{B}\left(\Xi^{0} \rightarrow \Lambda^{0} \pi^{0}\right)$. We obtain $\mathcal{B}\left(\Xi^{0} \rightarrow \Lambda^{0} \pi^{0}\right)=$ $(80.016 \pm 3.746) \%$, which is about $16 \%$ smaller than its data. The reason could be that the neglected $C_{+}$term or SU(3) breaking effects might give a contribution of a few percent to $\mathcal{B}\left(\Xi^{-} \rightarrow \Lambda^{0} \pi^{-}\right)$and $\mathcal{B}\left(\Xi^{0} \rightarrow \Lambda^{0} \pi^{0}\right)$.

\section{2. $T_{10} \rightarrow T_{8} M_{8}$ weak decays}

Feynman diagrams for the $T_{10} \rightarrow T_{8} M_{8}$ nonleptonic decays are also displayed in Fig. 3, and the SU(3) IRA amplitudes are

$$
\begin{aligned}
A\left(T_{10} \rightarrow T_{8} M_{8}\right)^{I R A}= & \bar{a}_{1} H(4)_{m}^{l k}\left(T_{10}\right)^{n i j}\left(T_{8}\right)_{[i k] j}\left(M_{8}\right)_{l}^{m}+\bar{a}_{2} H(4)_{m}^{l k}\left(T_{10}\right)^{n i j}\left(T_{8}\right)_{[j k] i}\left(M_{8}\right)_{l}^{m} \\
& +\bar{b}_{1} H(4)_{m}^{l k}\left(T_{10}\right)^{n i j}\left(T_{8}\right)_{[k]] i}\left(M_{8}\right)_{j}^{m}+\bar{b}_{2} H(4)_{m}^{l k}\left(T_{10}\right)^{n i j}\left(T_{8}\right)_{[k i] l}\left(M_{8}\right)_{j}^{m}+\bar{b}_{3} H(4)_{m}^{l k}\left(T_{10}\right)^{n i j}\left(T_{8}\right)_{[i l] k}\left(M_{8}\right)_{j}^{m} \\
& +\bar{c}_{1} H(4)_{j}^{l k}\left(T_{10}\right)^{n i j}\left(T_{8}\right)_{[k m] i}\left(M_{8}\right)_{l}^{m}+\bar{c}_{2} H(4)_{j}^{l k}\left(T_{10}\right)^{n i j}\left(T_{8}\right)_{[k i] m}\left(M_{8}\right)_{l}^{m}+\bar{c}_{3} H(4)_{j}^{l k}\left(T_{10}\right)^{n i j}\left(T_{8}\right)_{[i m] k}\left(M_{8}\right)_{j}^{m} \\
& +\bar{d}_{1} H(4)_{j}^{l k}\left(T_{10}\right)^{n i j}\left(T_{8}\right)_{[m k] l}\left(M_{8}\right)_{i}^{m}+\bar{d}_{2} H(4)_{j}^{l k}\left(T_{10}\right)^{n i j}\left(T_{8}\right)_{[m l] k}\left(M_{8}\right)_{i}^{m}+\bar{d}_{3} H(4)_{j}^{l k}\left(T_{10}\right)^{n i j}\left(T_{8}\right)_{[k l] m}\left(M_{8}\right)_{i}^{m} \\
& +\bar{e}_{1} H(\overline{2})^{k}\left(T_{10}\right)^{n i j}\left(T_{8}\right)_{[i m] j}\left(M_{8}\right)_{k}^{m}+\bar{e}_{2} H(\overline{2})^{k}\left(T_{10}\right)^{n i j}\left(T_{8}\right)_{[j m] i}\left(M_{8}\right)_{k}^{m} \\
& +\bar{f}_{1} H(\overline{2})^{k}\left(T_{10}\right)^{n i j}\left(T_{8}\right)_{[k i] m}\left(M_{8}\right)_{j}^{m}+\bar{f}_{2} H(\overline{2})^{k}\left(T_{10}\right)^{n i j}\left(T_{8}\right)_{[k m] i}\left(M_{8}\right)_{j}^{m}+\bar{f}_{3} H(\overline{2})^{k}\left(T_{10}\right)^{n i j}\left(T_{8}\right)_{[i m] k}\left(M_{8}\right)_{j}^{m} .
\end{aligned}
$$

Considering the fact that $H(4)_{m}^{l k}$ and $\left(T_{10}\right)^{n i j}$ are symmetric in upper indices, we have the relations

$$
\bar{a}_{2}=\bar{a}_{1}, \quad \bar{b}_{1}=0, \quad \bar{b}_{3}=-\bar{b}_{2}, \quad \bar{d}_{2}=\bar{d}_{1}, \quad \bar{d}_{3}=0, \quad \bar{e}_{2}=\bar{e}_{1} .
$$

Then Eq. (39) can be simplified as

$$
\begin{aligned}
A\left(T_{10} \rightarrow T_{8} M_{8}\right)^{I R A, J}= & \bar{a}_{1} H(4)_{m}^{l k}\left(T_{10}\right)^{n i j}\left(T_{8}\right)_{[i k] j}\left(M_{8}\right)_{l}^{m}+\bar{b}_{2} H(4)_{m}^{l k}\left(T_{10}\right)^{n i j}\left(T_{8}\right)_{[k i] l}\left(M_{8}\right)_{j}^{m} \\
& +\bar{c}_{1} H(4)_{j}^{l k}\left(T_{10}\right)^{n i j}\left(T_{8}\right)_{[k m] i}\left(M_{8}\right)_{l}^{m}+\bar{c}_{2} H(4)_{j}^{l k}\left(T_{10}\right)^{n i j}\left(T_{8}\right)_{[k i] m}\left(M_{8}\right)_{l}^{m} \\
& +\bar{c}_{3} H(4)_{j}^{l k}\left(T_{10}\right)^{n i j}\left(T_{8}\right)_{[i m] k}\left(M_{8}\right)_{j}^{m}+\bar{d}_{1} H(4)_{j}^{l k}\left(T_{10}\right)^{n i j}\left(T_{8}\right)_{[m k] l}\left(M_{8}\right)_{i}^{m} \\
& +\bar{e}_{1} H(\overline{2})^{k}\left(T_{10}\right)^{n i j}\left(T_{8}\right)_{[i m] j}\left(M_{8}\right)_{k}^{m}+\bar{f}_{1} H(\overline{2})^{k}\left(T_{10}\right)^{n i j}\left(T_{8}\right)_{[k i] m}\left(M_{8}\right)_{j}^{m} \\
& +\bar{f}_{2} H(\overline{2})^{k}\left(T_{10}\right)^{n i j}\left(T_{8}\right)_{[k m] i}\left(M_{8}\right)_{j}^{m}+\bar{f}_{3} H(\overline{2})^{k}\left(T_{10}\right)^{n i j}\left(T_{8}\right)_{[i m] k}\left(M_{8}\right)_{j}^{m} .
\end{aligned}
$$

The IRA amplitudes for the $T_{10} \rightarrow T_{8} P_{8}$ weak decays are listed in Table VIII, and the IRA amplitudes for the $T_{10} \rightarrow T_{8} V_{8}$ weak decays have similar relations. If neglecting the $H(\overline{4})_{2}^{22}$ terms and the $c_{i}$ terms in $H(4)_{1}^{12}$, and redefining the parameters 
TABLE VIII. The SU(3) IRA amplitudes of the $T_{10} \rightarrow T_{8} M_{8}$ weak decays.

\begin{tabular}{lcccc}
\hline \hline Amplitudes & $H(4)_{1}^{12}=\frac{1}{3}$ & $H(4)_{2}^{22}=-\frac{1}{3}$ & $H(\overline{2})^{2}=1$ & Simplified amplitudes \\
\hline$A\left(\Omega^{-} \rightarrow \Xi^{0} \pi^{-}\right)$ & $2 \bar{a}_{1}$ & & $2 \bar{e}_{1}$ & $\bar{A}_{1}$ \\
$\sqrt{2} A\left(\Omega^{-} \rightarrow \Xi^{-} \pi^{0}\right)$ & $-2 \bar{a}_{1}$ & $2 \bar{a}_{1}$ & $2 \bar{e}_{1}$ & $\bar{A}_{2}$ \\
$\sqrt{6} A\left(\Omega^{-} \rightarrow \Lambda^{0} K^{-}\right)$ & & & $\bar{f}_{1}+2 \bar{f}_{2}+\bar{f}_{3}$ & $\bar{A}_{3}$ \\
$3 \sqrt{2} A\left(\Xi^{*-} \rightarrow \Lambda^{0} \pi^{-}\right)$ & $6 \bar{a}_{1}$ & $2 \bar{c}_{1}+\bar{c}_{2}+\bar{c}_{3}$ & $6 \bar{e}_{1}+\bar{f}_{1}+2 \bar{f}_{2}+\bar{f}_{3}$ & $3 \bar{A}_{1}+\bar{A}_{3}$ \\
$\sqrt{6} A\left(\Xi^{*-} \rightarrow \Sigma^{0} \pi^{-}\right)$ & $2 \bar{a}_{1}-4 \bar{b}_{2}$ & $\bar{c}_{3}-\bar{c}_{2}$ & $2 \bar{e}_{1}-\bar{f}_{1}+\bar{f}_{3}$ & \\
$\sqrt{6} A\left(\Xi^{*-} \rightarrow \Sigma^{-} \pi^{0}\right)$ & $-2 \bar{a}_{1}$ & $2 \bar{a}_{1}-2 \bar{b}_{2}+\bar{c}_{3}-\bar{c}_{2}$ & $2 \bar{e}_{1}-\bar{f}_{1}+\bar{f}_{3}$ & \\
$\sqrt{3} A\left(\Xi^{* 0} \rightarrow \Sigma^{+} \pi^{-}\right)$ & $2 \bar{a}_{1}+\bar{c}_{3}-\bar{c}_{2}$ & & $2 \bar{e}_{1}$ & $\bar{A}_{1}$ \\
$\sqrt{3} A\left(\Xi^{* 0} \rightarrow \Sigma^{-} \pi^{+}\right)$ & $\bar{c}_{2}-\bar{c}_{3}$ & $2 \bar{b}_{2}$ & $\bar{f}_{1}-\bar{f}_{3}$ & \\
$6 A\left(\Xi^{* 0} \rightarrow \Lambda^{0} \pi^{0}\right)$ & $-6 \bar{a}_{1}+2\left(2 \bar{c}_{1}+\bar{c}_{2}+\bar{c}_{3}\right)$ & $6 \bar{a}_{1}$ & $6 \bar{e}_{1}+\bar{f}_{1}+2 \bar{f}_{2}+\bar{f}_{3}$ & $3 \bar{A}_{2}+\bar{A}_{3}$ \\
$2 \sqrt{3} A\left(\Xi^{* 0} \rightarrow \Sigma^{0} \pi^{0}\right)$ & $2 \bar{a}_{1}-4 \bar{b}_{2}$ & $-2 \bar{a}_{1}$ & $-2 \bar{e}_{1}-\bar{f}_{1}+\bar{f}_{3}$ & \\
$\sqrt{3} A\left(\Xi^{*-} \rightarrow n K^{-}\right)$ & $2 \bar{b}_{2}$ & $2 \bar{d}_{1}$ & $-\bar{f}_{2}-\bar{f}_{3}$ & \\
$\sqrt{3} A\left(\Sigma^{*-} \rightarrow n \pi^{-}\right)$ & $-2 \bar{a}_{1}+2 \bar{b}_{2}$ & $-\bar{c}_{1}-\bar{c}_{3}+2 \bar{d}_{1}$ & $-2 \bar{e}_{1}-\bar{f}_{2}-\bar{f}_{3}$ & \\
\hline \hline
\end{tabular}

$$
\begin{aligned}
& \bar{A}_{1}=2\left(\bar{a}_{1}+\bar{e}_{1}\right), \\
& \bar{A}_{2}=2\left(-\bar{a}_{1}+\bar{e}_{1}\right), \\
& \bar{A}_{3}=2\left(\bar{f}_{1}+2 \bar{f}_{2}+\bar{f}_{2}\right),
\end{aligned}
$$

the six decay amplitudes can be given in simpler forms, which are shown in the last column of Table VIII. Furthermore, we have the relation $A\left(\Xi^{*-} \rightarrow \Sigma^{0} \pi^{-}\right)=$ $A\left(\Xi^{*-} \rightarrow \Sigma^{-} \pi^{0}\right)$ if considering only the dominant $H(\overline{2})^{2}$ contributions.

The branching ratios of $T_{10} \rightarrow T_{8} P_{8}$ can be obtained in terms of the IRA amplitudes

$\mathcal{B}\left(T_{10 A} \rightarrow T_{8 B} P_{8}\right)=\frac{\tau_{A}\left|p_{c m}\right|}{16 \pi m_{A}^{2}}\left|A\left(T_{10 A} \rightarrow T_{8 B} P_{8}\right)\right|^{2}$,

and the mass difference in $A\left(T_{10 A} \rightarrow T_{8 B} P_{8}\right)$, which is similar to Eq. (36), is also considered.

At present, only three $\Omega^{-}$decay modes have been measured:

$$
\begin{aligned}
\mathcal{B}\left(\Omega^{-} \rightarrow \Xi^{0} \pi^{-}\right)\left(\times 10^{-2}\right) & =(23.6 \pm 0.7) \times 10^{-2}, \\
\mathcal{B}\left(\Omega^{-} \rightarrow \Xi^{-} \pi^{0}\right)\left(\times 10^{-2}\right) & =(8.6 \pm 0.4) \times 10^{-2}, \\
\mathcal{B}\left(\Omega^{-} \rightarrow \Lambda^{0} K^{-}\right)\left(\times 10^{-2}\right) & =(67.8 \pm 0.7) \times 10^{-2} .
\end{aligned}
$$

We obtain $\left|\bar{A}_{1}\right|=8.54 \pm 0.19,\left|\bar{A}_{2}\right|=7.47 \pm 0.23$, and $\left|\bar{A}_{3}\right|=5.36 \pm 0.08$ from the data of $\mathcal{B}\left(\Omega^{-} \rightarrow \Xi^{0} \pi^{-}\right)$, $\mathcal{B}\left(\Omega^{-} \rightarrow \Xi^{-} \pi^{0}\right)$, and $\mathcal{B}\left(\Omega^{-} \rightarrow \Lambda^{0} K^{-}\right)$, respectively. Then we predict that

$$
\begin{aligned}
\mathcal{B}\left(\Xi^{*-} \rightarrow \Lambda^{0} \pi^{-}\right) & =(1.06 \pm 0.90) \times 10^{-12}, \\
\mathcal{B}\left(\Xi^{* 0} \rightarrow \Sigma^{+} \pi^{-}\right) & =(5.96 \pm 0.58) \times 10^{-14}, \\
\mathcal{B}\left(\Xi^{* 0} \rightarrow \Lambda^{0} \pi^{0}\right) & =(5.02 \pm 4.06) \times 10^{-13},
\end{aligned}
$$

where the prediction for $\mathcal{B}\left(\Xi^{*-} \rightarrow \Lambda^{0} \pi^{-}\right)$depends on the relative phase between $\bar{A}_{1}$ and $\bar{A}_{3}$, and the prediction for
$\mathcal{B}\left(\Xi^{* 0} \rightarrow \Lambda^{0} \pi^{0}\right)$ depends on the relative phase between $\bar{A}_{2}$ and $\bar{A}_{3}$.

\section{B. Electromagnetic or strong decays of light baryons}

The light baryons $T_{10}$ can also decay through electromagnetic or strong interactions. The Feynman diagram of the electromagnetic or strong (ES) decays of $T_{10}$ is shown in Fig. 4. In this case, we need only consider the SU(3) symmetry between the initial and final states. The SU(3) IRA amplitude of the $T_{10} \rightarrow T_{8} M_{8}$ ES decay is

$$
A\left(T_{10} \rightarrow T_{8} M_{8}\right)^{\mathrm{ES}, \mathrm{IRA}}=\beta_{1}\left(T_{10}\right)^{i j k}\left(T_{8}\right)_{[i l] j}\left(M_{8}\right)_{k}^{l} .
$$

There is only one parameter $\beta_{1}$ for this IRA amplitude. The IRA amplitudes of all of the ES $T_{10} \rightarrow T_{8} P_{8}$ decays are given in Table IX.

For these ES decays, only three branching ratios are measured; they are given in Table X. We first get $\left|\beta_{1}\right|$ from the data of $\mathcal{B}\left(\Sigma^{*} \rightarrow \Sigma \pi\right)$, then also consider the experimental constraint from $\mathcal{B}\left(\Sigma^{*} \rightarrow \Lambda \pi\right)$, and finally give the

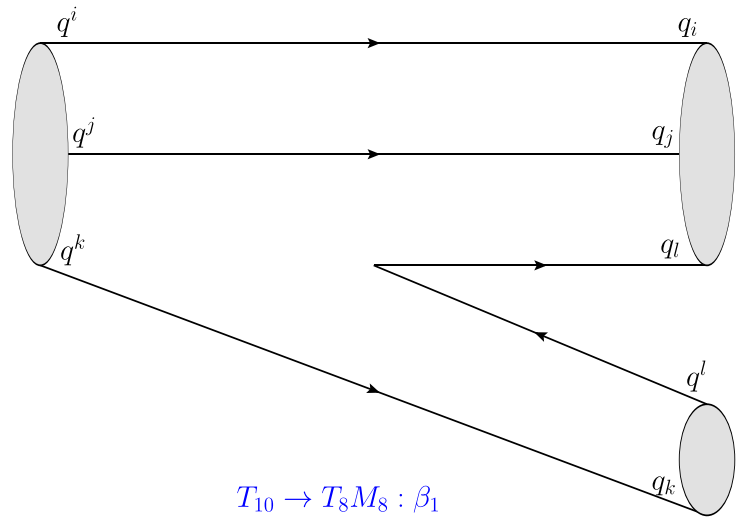

FIG. 4. Feynman diagram of the IRA for the $T_{10} \rightarrow T_{8} M_{8}$ ES decays. 
TABLE IX. The IRA amplitudes of the $T_{10} \rightarrow T_{8} P_{8}$ ES decays under the $\mathrm{SU}(3)$ flavor symmetry.

\begin{tabular}{lc}
\hline \hline Amplitudes & SU(3) IRA amplitudes \\
\hline$\sqrt{6} A\left(\Sigma^{*+} \rightarrow \Sigma^{0} \pi^{+}\right)$ & $\beta_{1}$ \\
$\sqrt{6} A\left(\Sigma^{*+} \rightarrow \Sigma^{+} \pi^{0}\right)$ & $\beta_{1}$ \\
$2 \sqrt{6} A\left(\Sigma^{* 0} \rightarrow \Sigma^{0} \pi^{0}\right)$ & 0 \\
$\sqrt{6} A\left(\Sigma^{* 0} \rightarrow \Sigma^{+} \pi^{-}\right)$ & $\beta_{1}$ \\
$\sqrt{6} A\left(\Sigma^{* 0} \rightarrow \Sigma^{-} \pi^{+}\right)$ & $-\beta_{1}$ \\
$\sqrt{6} A\left(\Sigma^{*-} \rightarrow \Sigma^{-} \pi^{0}\right)$ & $\beta_{1}$ \\
$\sqrt{6} A\left(\Sigma^{*-} \rightarrow \Sigma^{0} \pi^{-}\right)$ & $\beta_{1}$ \\
$3 \sqrt{2} A\left(\Sigma^{*+} \rightarrow \Lambda^{0} \pi^{+}\right)$ & $-3 \beta_{1}$ \\
$6 \sqrt{2} A\left(\Sigma^{* 0} \rightarrow \Lambda^{0} \pi^{0}\right)$ & $6 \beta_{1}$ \\
$3 \sqrt{2} A\left(\Sigma^{*-} \rightarrow \Lambda^{0} \pi^{-}\right)$ & $3 \beta_{1}$ \\
$\sqrt{6} A\left(\Xi^{* 0} \rightarrow \Xi^{0} \pi^{0}\right)$ & $\beta_{1}$ \\
$\sqrt{3} A\left(\Xi^{* 0} \rightarrow \Xi^{-} \pi^{+}\right)$ & $-\beta_{1}$ \\
$\sqrt{6} A\left(\Xi^{*-} \rightarrow \Xi^{-} \pi^{0}\right)$ & $\beta_{1}$ \\
$\sqrt{3} A\left(\Xi^{*-} \rightarrow \Xi^{0} \pi^{-}\right)$ & $\beta_{1}$ \\
\hline \hline
\end{tabular}

predictions of other specific branching ratios. Our SU(3) IRA predictions are given in Table $X$, where one can see that, within $1 \sigma$ error, the experimental result of $\mathcal{B}\left(\Sigma^{*} \rightarrow\right.$ $\Lambda \pi$ ) can effectively constrain $\left|\beta_{1}\right|$. In addition, when the IRA predictions are consistent with the data of $\mathcal{B}\left(\Sigma^{*} \rightarrow \Sigma \pi\right)$ and $\mathcal{B}\left(\Sigma^{*} \rightarrow \Lambda \pi\right)$, the prediction of $\mathcal{B}\left(\Xi^{*} \rightarrow \Xi \pi\right)$ is slightly larger than its experimental result, which might imply that the SU(3) breaking effects could give visible contributions to $\mathcal{B}\left(\Xi^{*} \rightarrow \Xi \pi\right)$. Nevertheless, the prediction and

TABLE X. Branching ratios of $T_{10} \rightarrow T_{8} P_{8}$ ES decays within $1 \sigma$ error.

\begin{tabular}{lcc}
\hline \hline Branching ratios & Experimental Data & SU(3) IRA \\
\hline $\mathcal{B}\left(\Sigma^{*+} \rightarrow \Sigma^{0} \pi^{+}\right)\left(\times 10^{-2}\right)$ & $\ldots$ & $5.34 \pm 0.50$ \\
$\mathcal{B}\left(\Sigma^{*+} \rightarrow \Sigma^{+} \pi^{0}\right)\left(\times 10^{-2}\right)$ & $\ldots$ & $6.59 \pm 0.61$ \\
$\mathcal{B}\left(\Sigma^{* 0} \rightarrow \Sigma^{0} \pi^{0}\right)\left(\times 10^{-2}\right)$ & $\ldots$ & 0 \\
$\mathcal{B}\left(\Sigma^{* 0} \rightarrow \Sigma^{+} \pi^{-}\right)\left(\times 10^{-2}\right)$ & $\ldots$ & $6.20 \pm 0.78$ \\
$\mathcal{B}\left(\Sigma^{* 0} \rightarrow \Sigma^{-} \pi^{+}\right)\left(\times 10^{-2}\right)$ & $\ldots$ & $4.71 \pm 0.59$ \\
$\mathcal{B}\left(\Sigma^{*-} \rightarrow \Sigma^{-} \pi^{0}\right)\left(\times 10^{-2}\right)$ & $\ldots$ & $5.40 \pm 0.60$ \\
$\mathcal{B}\left(\Sigma^{*-} \rightarrow \Sigma^{0} \pi^{-}\right)\left(\times 10^{-2}\right)$ & $\ldots$ & $5.66 \pm 0.63$ \\
$\mathcal{B}\left(\Sigma^{*} \rightarrow \Sigma \pi\right)\left(\times 10^{-2}\right)$ & $11.7 \pm 1.5$ & $11.24 \pm 0.28$ \\
$\mathcal{B}\left(\Sigma^{*+} \rightarrow \Lambda^{0} \pi^{+}\right)\left(\times 10^{-2}\right)$ & $\ldots$ & $86.14 \pm 7.62$ \\
$\mathcal{B}\left(\Sigma^{* 0} \rightarrow \Lambda^{0} \pi^{0}\right)\left(\times 10^{-2}\right)$ & $\ldots$ & $91.68 \pm 11.36$ \\
$\mathcal{B}\left(\Sigma^{*-} \rightarrow \Lambda^{0} \pi^{-}\right)\left(\times 10^{-2}\right)$ & $\ldots$ & $84.44 \pm 8.96$ \\
$\mathcal{B}\left(\Sigma^{*} \rightarrow \Lambda^{0} \pi\right)\left(\times 10^{-2}\right)$ & $87.0 \pm 1.5$ & $87.00 \pm 1.50^{\mathrm{a}}$ \\
$\mathcal{B}\left(\Xi^{* 0} \rightarrow \Xi^{0} \pi^{0}\right)\left(\times 10^{-2}\right)$ & $\ldots$ & $48.22 \pm 6.55$ \\
$\mathcal{B}\left(\Xi^{* 0} \rightarrow \Xi^{-} \pi^{+}\right)\left(\times 10^{-2}\right)$ & $\ldots$ & $76.23 \pm 10.32$ \\
$\mathcal{B}\left(\Xi^{*-} \rightarrow \Xi^{-} \pi^{0}\right)\left(\times 10^{-2}\right)$ & $\ldots$ & $43.05 \pm 11.01$ \\
$\mathcal{B}\left(\Xi^{*-} \rightarrow \Xi^{0} \pi^{-}\right)\left(\times 10^{-2}\right)$ & $\ldots$ & $94.33 \pm 24.12$ \\
$\mathcal{B}\left(\Xi^{*} \rightarrow \Xi \pi\right)\left(\times 10^{-2}\right)$ & 100 & $131.01 \pm 24.40^{\mathrm{b}}$ \\
\hline \hline
\end{tabular}

${ }^{\mathrm{a}}$ Experimental data have been used to give the effective constraints on the parameters.

${ }^{b}$ Experimental data have not been used to constrain the parameters. experimental data of $\mathcal{B}\left(\Xi^{*} \rightarrow \Xi \pi\right)$ can be consistent within $1.3 \sigma$ error. And moreover, the decay width predictions of $\Xi^{* 0} \rightarrow \Xi \pi$ and $\Xi^{*-} \rightarrow \Xi \pi$ in the chiral quark-soliton model are also slightly larger than their experimental data [67].

Note that the ES $T_{8} \rightarrow T_{8} P_{8}$ decays and the ES $T_{10} \rightarrow$ $T_{8} K$ decays are not allowed by the phase space since the sum of the final hadron masses is larger than the mass of the initial state.

\section{SUMMARY}

Light baryon decays play a very important role in testing of the SM and searching for new physics beyond the SM. Many decay modes have been measured, and some decays can be studied at BESIII and LHCb experiments now. Motivated by this, we have analyzed the semileptonic decays and two-body nonleptonic decays of light baryon octets and decuplets by using the irreducible representation approach to test the SU(3) flavor symmetry. Our main results can be summarized as follows:

(a) Semileptonic light baryon decays.-We have found that all branching ratio predictions of octet and decuplet baryons through $s \rightarrow u \ell^{-} \bar{\nu}_{\ell}$ and $d \rightarrow$ $u e^{-} \bar{\nu}_{e}$ transitions with SU(3) IRA in the $S_{2}$ case are quite consistent with the present experimental measurements within $1 \sigma$ error. We have predicted that $\mathcal{B}\left(\Xi^{-} \rightarrow \Sigma^{0} \mu^{-} \bar{\nu}_{\mu}\right)$ and $\mathcal{B}\left(\Omega^{-} \rightarrow \Xi^{0} \mu^{-} \bar{\nu}_{\mu}\right)$ are on the order of magnitudes of $10^{-6}$ and $10^{-3}$, respectively, and that $\mathcal{B}\left(\Sigma^{-} \rightarrow \Sigma^{0} e^{-} \bar{\nu}_{e}, \Xi^{-} \rightarrow \Xi^{0} e^{-} \bar{\nu}_{e}\right)$ are on the order of $10^{-10}$. These decays promise to be observed by the BESIII and LHCb experiments or other future experiments. However, other branching ratios, which are in the range of $10^{-20}$ to $10^{-13}$, may not be measured for a long time. Moreover, the longitudinal branching ratios of decays of $T_{8 A} \rightarrow T_{8 B} \ell^{-} \bar{\nu}_{\ell}$ have also been predicted in this work.

(b) Nonleptonic two-body light baryon decays.-We have obtained the relations of different decay amplitudes by the SU(3) IRA and isospin symmetry. In $T_{8} \rightarrow T_{8} P_{8}$ weak decays, we have found that SU(3) IRA predictions of the branching ratios of $\Sigma, \Lambda$ baryons are consistent with the present experimental data, $\mathcal{B}\left(\Sigma^{0} \rightarrow p \pi^{-}, n \pi^{0}\right)$ are on the order of $10^{-10}$ by the SU(3) IRA or isospin symmetry, and the neglected $C_{+}$terms or SU(3) symmetry breaking effects might give a contribution of a few percent to the two branching ratios of $\Xi \rightarrow \Lambda \pi$. In the $T_{10} \rightarrow T_{8} P$ weak decays, we have predicted that $\mathcal{B}\left(\Xi^{*-} \rightarrow \Lambda^{0} \pi^{-}\right), \quad \mathcal{B}\left(\Xi^{* 0} \rightarrow \Lambda^{0} \pi^{0}\right), \quad$ and $\quad \mathcal{B}\left(\Xi^{* 0} \rightarrow\right.$ $\left.\Sigma^{+} \pi^{-}\right)$are on the order of $10^{-12}, 10^{-13}$, and $10^{-14}$, respectively. In the $T_{10} \rightarrow T_{8} P_{8}$ ES decays, when the IRA predictions are consistent with the data of $\mathcal{B}\left(\Sigma^{*} \rightarrow \Sigma \pi\right)$ and $\mathcal{B}\left(\Sigma^{*} \rightarrow \Lambda \pi\right)$, the prediction of $\mathcal{B}\left(\Xi^{*} \rightarrow \Xi \pi\right)$ is slightly larger than the experimental data, which implies that the SU(3) symmetry 
breaking effects could give visible contributions to $\mathcal{B}\left(\Xi^{*} \rightarrow \Xi \pi\right)$. In addition, we have given all of the specific branching ratio predictions for these $T_{10} \rightarrow$ $T_{8} P_{8}$ ES decays.

Although flavor $\mathrm{SU}(3)$ symmetry is approximate, it can still provide us with very useful information about these decays. According to our predictions, some branching ratios will be accessible to the experiments at BESIII and $\mathrm{LHCb}$. Our results in this work could be used to test the $\mathrm{SU}(3)$ flavor symmetry approach in light baryon decays in future experiments.

\section{ACKNOWLEDGMENTS}

R.-M. W. thanks Wei Wang for the helpful communications. This work was supported by the National Natural Science Foundation of China (Contracts No. 11675137, No. 11875168, and No. 11875054); the Joint LargeScale Scientific Facility Funds of the NSFC and CAS under Contract No. U1532257; the CAS under Contract No. QYZDJ-SSWSLH003; and the National Key Basic Research Program of China under Contract No. 2015CB856700.
[1] M. Tanabashi et al. (Particle Data Group), Phys. Rev. D 98, 030001 (2018).

[2] H.-B. Li, Front. Phys. 12, 121301 (2017); 14, 64001(E) (2019).

[3] I. I. Bigi, X. W. Kang, and H. B. Li, Chin. Phys. C 42, 013101 (2018).

[4] D. M. Asner et al., Int. J. Mod. Phys. A 24, 499 (2009).

[5] M. Ablikim et al. (BESIII Collaboration), Nat. Phys. 15, 631 (2019).

[6] R. Aaij et al. (LHCb Collaboration), Phys. Rev. Lett. 120, 221803 (2018).

[7] A. A. Alves Junior et al., J. High Energy Phys. 05 (2019) 048.

[8] S. Weinberg, J. Phys. Conf. Ser. 196, 012002 (2009).

[9] N. Severijns, M. Beck, and O. Naviliat-Cuncic, Rev. Mod. Phys. 78, 991 (2006).

[10] N. Cabibbo, Phys. Rev. Lett. 10, 531 (1963).

[11] V. Cirigliano, M. Gonzalez-Alonso, and M. L. Graesser, J. High Energy Phys. 02 (2013) 046.

[12] H. M. Chang, M. González-Alonso, and J. Martin Camalich, Phys. Rev. Lett. 114, 161802 (2015).

[13] X. G. He, Eur. Phys. J. C 9, 443 (1999).

[14] X. G. He, Y. K. Hsiao, J. Q. Shi, Y. L. Wu, and Y. F. Zhou, Phys. Rev. D 64, 034002 (2001).

[15] B. Grinstein and R. F. Lebed, Phys. Rev. D 53, 6344 (1996).

[16] B. Grinstein, D. Pirtskhalava, D. Stone, and P. Uttayarat, Phys. Rev. D 89, 114014 (2014).

[17] H. K. Fu, X. G. He, and Y. K. Hsiao, Phys. Rev. D 69, 074002 (2004).

[18] Y. K. Hsiao, C. F. Chang, and X. G. He, Phys. Rev. D 93, 114002 (2016).

[19] X. G. He and G. N. Li, Phys. Lett. B 750, 82 (2015).

[20] M. Gronau, O. F. Hernandez, D. London, and J. L. Rosner, Phys. Rev. D 50, 4529 (1994).

[21] M. Gronau, O. F. Hernandez, D. London, and J. L. Rosner, Phys. Rev. D 52, 6356 (1995).

[22] S. H. Zhou, Q. A. Zhang, W. R. Lyu, and C. D. L, Eur. Phys. J. C 77, 125 (2017).

[23] H. Y. Cheng, C. W. Chiang, and A. L. Kuo, Phys. Rev. D 91, 014011 (2015).

[24] M. He, X. G. He, and G. N. Li, Phys. Rev. D 92, 036010 (2015).
[25] N. G. Deshpande and X. G. He, Phys. Rev. Lett. 75, 1703 (1995).

[26] S. Shivashankara, W. Wu, and A. Datta, Phys. Rev. D 91, 115003 (2015).

[27] Y. Grossman and D. J. Robinson, J. High Energy Phys. 04 (2013) 067.

[28] D. Pirtskhalava and P. Uttayarat, Phys. Lett. B 712, 81 (2012).

[29] H. Y. Cheng and C. W. Chiang, Phys. Rev. D 86, 014014 (2012).

[30] M. J. Savage and R. P. Springer, Phys. Rev. D 42, 1527 (1990).

[31] M. J. Savage, Phys. Lett. B 257, 414 (1991).

[32] G. Altarelli, N. Cabibbo, and L. Maiani, Phys. Lett. 57B, 277 (1975).

[33] C.-D. Lü, W. Wang, and F.-S. Yu, Phys. Rev. D 93, 056008 (2016).

[34] C. Q. Geng, Y. K. Hsiao, Y. H. Lin, and L. L. Liu, Phys. Lett. B 776, 265 (2018).

[35] C. Q. Geng, Y. K. Hsiao, C. W. Liu, and T. H. Tsai, Phys. Rev. D 97, 073006 (2018).

[36] C. Q. Geng, Y. K. Hsiao, C. W. Liu, and T. H. Tsai, J. High Energy Phys. 11 (2017) 147.

[37] C.-Q. Geng, C.-W. Liu, T.-H. Tsai, and S.-W. Yeh, Phys. Lett. B 792, 214 (2019).

[38] W. Wang, Z. P. Xing, and J. Xu, Eur. Phys. J. C 77, 800 (2017).

[39] D. Wang, Eur. Phys. J. C 79, 429 (2019).

[40] D. Wang, P. F. Guo, W. H. Long, and F. S. Yu, J. High Energy Phys. 03 (2018) 066.

[41] S. Müller, U. Nierste, and S. Schacht, Phys. Rev. D 92, 014004 (2015).

[42] G. Hiller, M. Jung, and S. Schacht, Phys. Rev. D 87, 014024 (2013).

[43] M. Gronau, Phys. Rev. D 91, 076007 (2015).

[44] J. M. Gaillard and G. Sauvage, Annu. Rev. Nucl. Part. Sci. 34, 351 (1984).

[45] T. N. Pham, Phys. Rev. D 87, 016002 (2013).

[46] M. E. Carrillo-Serrano, I. C. Cloet, and A. W. Thomas, Phys. Rev. C 90, 064316 (2014).

[47] R. Flores-Mendieta, E. E. Jenkins, and A. V. Manohar, Phys. Rev. D 58, 094028 (1998). 
[48] A. Kadeer, J. G. Korner, and U. Moosbrugger, Eur. Phys. J. C 59, 27 (2009).

[49] S. Sasaki, Phys. Rev. D 96, 074509 (2017).

[50] S. Sasaki, Phys. Rev. D 86, 114502 (2012).

[51] S. Sasaki and T. Yamazaki, Phys. Rev. D 79, 074508 (2009).

[52] G. Villadoro, Phys. Rev. D 74, 014018 (2006).

[53] A. Lacour, B. Kubis, and U. G. Meissner, J. High Energy Phys. 10 (2007) 083.

[54] A. Faessler, T. Gutsche, B. R. Holstein, M. A. Ivanov, J. G. Korner, and V. E. Lyubovitskij, Phys. Rev. D 78, 094005 (2008).

[55] D. Guadagnoli, V. Lubicz, M. Papinutto, and S. Simula, Nucl. Phys. B761, 63 (2007).

[56] N. Cabibbo, E. C. Swallow, and R. Winston, Annu. Rev. Nucl. Part. Sci. 53, 39 (2003).
[57] T. Ledwig, A. Silva, H. C. Kim, and K. Goeke, J. High Energy Phys. 07 (2008) 132.

[58] A. Faessler, T. Gutsche, B. R. Holstein, and V.E. Lyubovitskij, Phys. Rev. D 77, 114007 (2008).

[59] G. S. Yang and H. C. Kim, Phys. Rev. C 92, 035206 (2015).

[60] G. Buchalla, A. J. Buras, and M. E. Lautenbacher, Rev. Mod. Phys. 68, 1125 (1996).

[61] E. E. Jenkins, Nucl. Phys. B375, 561 (1992).

[62] A. Abd El-Hady and J. Tandean, Phys. Rev. D 61, 114014 (2000).

[63] R. Flores-Mendieta, Phys. Rev. D 99, 094033 (2019).

[64] J. Tandean and G. Valencia, Phys. Rev. D 67, 056001 (2003).

[65] B. Borasoy and E. Marco, Phys. Rev. D 67, 114016 (2003).

[66] X.-G. He, Y.-J. Shi, and W. Wang, arXiv:1811.03480.

[67] G. S. Yang and H. C. Kim, Phys. Lett. B 785, 434 (2018). 